\title{
Adaptive spatial combining for passive time-reversed communications $^{\text {a) }}$
}

\author{
João Gomes ${ }^{\text {b) }}$ \\ Institute for Systems and Robotics, Instituto Superior Técnico, 1049-001 Lisboa, Portugal \\ António Silva ${ }^{\mathrm{c})}$ and Sérgio Jesus ${ }^{\mathrm{d})}$ \\ Institute for Systems and Robotics, Universidade do Algarve, Campus de Gambelas, \\ 8005-139 Faro, Portugal
}

(Received 5 April 2007; revised 27 May 2008; accepted 28 May 2008)

Passive time reversal has aroused considerable interest in underwater communications as a computationally inexpensive means of mitigating the intersymbol interference introduced by the channel using a receiver array. In this paper the basic technique is extended by adaptively weighting sensor contributions to partially compensate for degraded focusing due to mismatch between the assumed and actual medium impulse responses. Two algorithms are proposed, one of which restores constructive interference between sensors, and the other one minimizes the output residual as in widely used equalization schemes. These are compared with plain time reversal and variants that employ postequalization and channel tracking. They are shown to improve the residual error and temporal stability of basic time reversal with very little added complexity. Results are presented for data collected in a passive time-reversal experiment that was conducted during the MREA'04 sea trial. In that experiment a single acoustic projector generated a 2/4-PSK (phase-shift keyed) stream at $200 / 400$ baud, modulated at $3.6 \mathrm{kHz}$, and received at a range of about $2 \mathrm{~km}$ on a sparse vertical array with eight hydrophones. The data were found to exhibit significant Doppler scaling, and a resampling-based preprocessing method is also proposed here to compensate for that scaling.

(C) 2008 Acoustical Society of America. [DOI: 10.1121/1.2946711]

PACS number(s): 43.60.Dh, 43.60.Tj, 43.60.Gk, 43.60.Fg [DRD] Pages: 1038-1053

\section{INTRODUCTION}

Time reversal is a wave backpropagation technique that cleverly exploits the reciprocity of linear wave propagation to concentrate signals at desired points in a waveguide with little knowledge about the medium. ${ }^{1}$ The potential of time reversal for underwater communications was recognized and attracted much attention after the practical feasibility of this technique was demonstrated in the ocean. ${ }^{2}$

Active time-reversed (TR) focusing is achieved by transmitting a channel probe from the intended focal spot to an array of transducers that sample the incoming pressure field. These signals are then reversed in time and retransmitted, creating a replica field that converges on the original source location and approximately regenerates the initial wave form, undoing much of the delay dispersion caused by multipath. Due to its peculiar mode of operation, this type of source/receiver array is often referred to as a time-reversal mirror (TRM). When the principle of time reversal is applied to digital communications, the measured probe source ping is modulated with an information-bearing wave form, which can then be demodulated at the focus with relatively low algorithmic complexity. Passive time reversal, or passive phase conjugation ${ }^{3}$ (PPC), is conceptually similar to the

\footnotetext{
${ }^{\mathrm{a}}$ Portions of this work were presented at the MTS/IEEE Oceans'06 Conference, Boston, MA, September 2006.

${ }^{b)}$ Electronic mail: jpg@isr.ist.utl.pt

${ }^{c)}$ Electronic mail: asilva@ualg.pt

d)Electronic mail: sjesus@ualg.pt
}

above technique, yet both the probe and message are sequentially sent from the source, so the array only operates in receive mode. Focusing is performed synthetically at the array by convolving the time-reversed distorted probes with received data packets. ${ }^{3}$ This is, in fact, a multichannel combining (MC) strategy ${ }^{4}$ whose parameters are directly measured from the data, not derived by optimizing a cost function.

Time reversal finds applications in diverse areas such as optics, materials testing, imaging, and medicine. ${ }^{5}$ In underwater acoustics, digital communications have provided the backdrop for many published applications of this technique. In fact, the wave form regeneration property of TRM is highly relevant in underwater acoustic communications, where intersymbol interference (ISI) caused by multipath is usually the single most important distortion to be compensated. ${ }^{4}$ Stojanovic ${ }^{6}$ provides an overview of much of the research work in this area.

Both active $e^{7,8}$ and passive ${ }^{9-11}$ TR communications have been demonstrated in the ocean, the latter being more popular due to a simpler hardware setup. These experimental results, and other theoretical analyses, ${ }^{6,12,13}$ suggest that time reversal by itself will not ensure reliable detection of the transmitted symbols, and should be complemented by adaptive equalization at the receiver to remove the residual ISI and compensate for channel variations. Arguably, the overall reduction in computational complexity at the receiver af- 
forded by the integration of time reversal into an acoustic link more than makes up for the moderate degradation in performance.

Most of the experiments reported to date employ singlecarrier coherent signaling, although time reversal can easily be adapted to other modulations as well. ${ }^{14}$ In fact, in line with a popular trend in underwater communications, several techniques first developed in wireless terrestrial communications have been investigated and proposed for acoustic links based on time reversal. This trend continues with multipleinput/multiple-output communications, which have provided large performance improvements in wireless radio and show great promise in underwater communications. ${ }^{15}$

In the methods that have been proposed so far for simultaneous equalization and time reversal the two systems are operated in tandem, i.e., a TRM creates a single-channel signal which is then independently processed by an equalizer. ${ }^{6,12,16}$ In PPC, however, the signals received at an array of hydrophones are synthetically combined after convolving them with estimates of the (reversed) channel impulse responses. This provides increased flexibility relative to active TR, as these signals may be individually postprocessed prior to generating a single-channel wave form.

This paper examines low-complexity PPC approaches where a single combining coefficient is used per array sensor to improve upon the performance of basic PPC under channel variations. These coefficients are adjusted at each symbol interval by either iteratively minimizing the output meansquare error (MSE) or maximizing the output magnitude. This approach is motivated by the observation that poor signal-to-interference (ISI+noise) ratio that occurs due to environment mismatch between the probe and packet transmissions can often be attributed to partially destructive interference between contributions from different hydrophone signals, in spite of appropriate temporal alignment. Note that the proposed structures are actually very short multichannel equalizers, and one could envisage using more elaborate filters as in conventional equalizers.

Environment mismatch is also addressed in decisiondirected PPC $^{17}$ (DDPPC) by tracking the channel impulse response continuously throughout a data packet to virtually eliminate the delay between probe capture and filtering. It should be emphasized that the MC methods proposed here are simpler, as the probe is only captured during the packet preamble, and subsequently only one coefficient per sensor is tracked. By contrast, DDPPC must propagate a fully adaptive model of the channel response measured in each sensor (or at least the portion of it with higher energy and greater temporal stability), which is not necessarily less computationally demanding than conventional equalization schemes.

Results from a passive time-reversal experiment conducted off the west coast of Portugal during the MREA'04 sea trial are presented. A single acoustic projector generated a 2/4-PSK stream at 200 and 400 baud around a carrier frequency of $3.6 \mathrm{kHz}$, and the signals were received at a range of about $2 \mathrm{~km}$ on a vertical array with eight unevenly spaced hydrophones. The channel end points were in motion during this experiment, inducing significant Doppler scaling in the observed wave forms that could degrade the perfor- mance of TR focusing if left uncompensated. ${ }^{11}$ A broadband Doppler compensation method is proposed here, theoretically analyzed, and shown to perform very effectively in practice. The method itself is simple and similar ideas have been proposed in the past, but to the best of our knowledge no prior analysis of Doppler compensation on TRM performance has been published. In addition to documenting the performance of proposed MC algorithms, this work also aims to provide experimental results on various aspects of channel characterization, conventional equalization, and plain time reversal.

The paper is organized as follows. Section II introduces the signal model used for time reversal and describes the Doppler compensation method. Section III presents the multichannel combining algorithms, illustrates their performance in a simulated scenario, and discusses the synchronization and normalization postprocessing steps that are required to estimate data symbols from the TRM output. Section IV describes the MREA'04 sea trial and presents experimental results. The proposed MC algorithms are characterized and compared with plain multichannel equalization, plain TRM, simultaneous TRM and single-channel equalization, and DDPPC. Channel/probe estimation issues are also considered. Finally, Sec. V summarizes the main results, draws some conclusions, and suggests future research.

\section{MODELING OF TIME REVERSAL}

This section presents the notation used in the sequel for coherent communication using time reversal. The reader is referred to Refs. 1 and 2 for an overview of (narrowband) TR theory, and to several references on (broadband) TR communications $^{8,9,11}$ for lengthier discussions on specific aspects of data transmission. Throughout the paper convolution is denoted by the binary operator $*$ and complex conjugation by the superscript $(\cdot)^{*}$.

As is usually done in the context of bandwidth-efficient coherent communications, a complex representation in terms of baseband equivalent signals (i.e., complex envelopes) will henceforth be adopted for the real passband wave forms that are actually transmitted and received. ${ }^{18}$ Time reversal of bandpass signals must then be replaced by time reversal and conjugation of complex envelopes, but other than that all equations describing the self-focusing property remain unchanged.

Let $p(t)$ represent the ideal basic pulse shape of the modulated wave forms that are exchanged between the source and the TRM, which can also act as a convenient channel probe. Other wave forms used for channel identification, such as linear frequency modulated pulses, are also appropriate. Denoting by $g_{m}(t)$ the impulse response between the focal point and the $m$ th sensor of an $M$-element array, the distorted received probe is $h_{m}(t)=p(t) * g_{m}(t)$. In active time reversal the TRM then transmits an arbitrary data packet with pulse shape $h^{*}(-t)$ which, by virtue of TR focusing, will be approximately regenerated at the focal spot with the original pulse shape $p^{*}(-t)$. In passive phase conjugation the probe is followed by a data packet transmitted by the same source after a guard interval. Coherent single-carrier modu- 
lation is assumed throughout this work, such that the received signal component at the $m$ th sensor is given by

$$
y_{m}(t)=\sum_{k} a(k) h_{m}\left(t-k T_{b}\right) .
$$

In the complex baseband representation underlying Eq. (1) the information symbols $\{a(k)\}$, transmitted with interval $T_{b}$, belong to a discrete signal constellation. ${ }^{18}$ This is defined as a finite set of points in the complex plane that represent groups of bits from a digital message. Physically, the real and imaginary components of $a(k)$ are used for amplitude modulation of the in-phase and quadrature carriers when generating real bandpass wave forms. The symbols $\{a(k)\}$ are assumed to be uncorrelated random variables with zero mean and unit variance. The noise component will be ignored in the characterization of time reversal given below.

A plain passive mirror emulates active time reversal synthetically in a receive-only array. Its output, $z(t)$, is obtained by convolving the received packet (1) with the TR probe replica to generate a match-filtered signal in each sensor, $z_{m}(t)$, and then adding all contributions

$$
\begin{aligned}
& z_{m}(t)=h_{m}^{*}(-t) * y_{m}(t), \\
& z(t)=\sum_{m=1}^{M} z_{m}(t)=\sum_{k} a(k) q\left(t-k T_{b}\right) .
\end{aligned}
$$

In Eq. (3) $q(t)$ denotes the sum of temporal autocorrelations of received pulse shapes, sometimes referred to as the $q$-function ${ }^{19}(\mathrm{QF})$. In a static ocean environment it is related to the medium impulse responses as

$$
q(t)=\sum_{m=1}^{M} h_{m}^{*}(-t) * h_{m}(t)=r(t) * \gamma(t),
$$

where

$$
r(t)=p^{*}(-t) * p(t), \quad \gamma(t)=\sum_{m=1}^{M} g_{m}^{*}(-t) * g_{m}(t) .
$$

The multipath self-compensation property of time reversal implies that the spectrum of $\gamma(t)$ should be approximately constant across the bandwidth of $p(t)$ [and $r(t)]$, so that $q(t) \propto r(t)$ and an undistorted modulated wave form is regenerated. In practice a delay is introduced to ensure causality of the time-reversed probe in Eq. (2), and all operations are performed in $L$-oversampled discrete-time signals $y_{m}(n)$ $\triangleq y_{m}\left(n T_{b} / L\right)$ and $h_{m}(n)$.

Decoding is particularly simple when $p(t)$ has a root raised-cosine shape because then $r(t)$ in Eqs. (3) and (4) is a Nyquist pulse. ${ }^{18}$ Out-of-band noise removal can be accomplished by actually transmitting fourth-root raised-cosine signaling pulses ${ }^{10} s(t)$ such that $p(t)=s^{*}(-t) * s(t)$, and then prefiltering all received wave forms (probes and packets) by $s^{*}(-t)$ to reject noise and attain the desired equivalent pulse shape $p(t)$. To avoid unnecessarily complicating our notation we assume that $h_{m}$ and $y_{m}$ in Eqs. (1), (2), and (4) have already undergone filtering by $s^{*}(-t)$. The spectra of $p(t)$, $r(t)$, and $s(t)$ are then related by

$$
P(\omega)=R^{1 / 2}(\omega), \quad S(\omega)=R^{1 / 4}(\omega), \quad R(\omega) \geq 0 .
$$

Coherence issues. When channel variations occur between the probe and packet transmissions, autocorrelations in Eq. (4) are replaced by crosscorrelations between received pulses at different instants. This decreases the TRM's focusing power by degrading the impulselike behavior of $q(t)$.

To reduce the latency and mismatch between probe measuring and focusing, it is possible to discard the actual probe transmission and estimate it directly from a known preamble in the data packet. ${ }^{16}$ This has the added benefit of reducing the additive noise component in the probe estimate $h_{m}(t)$, which generates undesirable convolutional noise during focusing. In a nonstatic environment it may also filter out the contributions from paths with poor temporal coherence, thus reducing the jitter in match-filtered outputs. This idea is taken further in DDPPC, ${ }^{17}$ where the channel is tracked throughout data packets. A sharp QF is thus preserved even with very long packets because a low-latency channel estimate is always available.

The total number of parameters to be estimated by DDPPC in typical (multiple-hydrophone) discrete tap-delayline models of underwater channels may be quite large and impose a significant computational burden. Alternative strategies that perform simpler adaptation and require fewer parameters, such as the ones addressed in this paper, may therefore be of interest. While Flynn et al. ${ }^{17}$ argue in favor of iterative block least-squares estimation, in this work we adopt the exponentially windowed recursive least-squares (RLS) algorithm for channel estimation and tracking, which has been extensively used in underwater channel equalization and identification.

\section{A. Doppler distortion and compensation}

By decomposing a source with arbitrary space-time dependence as a superposition of monochromatic point sources, TR focusing may be shown to hold even for moving sources. ${ }^{1}$ This work addresses a restricted case where the source is assumed to be moving slowly enough over a sufficiently short period so that the medium impulse responses linking it to the array transducers remain approximately constant. TR experiments suggest that this hypothesis is more plausible for predominantly horizontal motion, as the size of the focal spot in the horizontal plane is larger than along the depth axis.

Given a nominal transmitted passband wave form with carrier frequency $\omega_{c}, \quad \widetilde{x}(t)=\operatorname{Re}\left\{x(t) e^{j \omega_{c} t}\right\}$, the equivalent Doppler-distorted transmission over a single path is

$$
\tilde{x}((1+\beta) t)=\operatorname{Re}\left\{x((1+\beta) t) e^{j \omega_{c} \beta t} e^{j \omega_{c} t}\right\},
$$

where $\beta$ is the time compression/dilation factor. For a moving transmitter with velocity $v$ heading toward a static receiver in a medium with sound speed $c \gg v, \beta$ is given by ${ }^{20}$

$$
\beta=\frac{1}{1-v / c}-1 \approx \frac{v}{c} .
$$

In terms of baseband signals, Eq. (7) amounts to time scaling of the original $x(t)$ and multiplication by a complex exponential with angular frequency $\omega_{c} \beta$. In a multipath environment 
several delayed contributions of the above type are observed at the receiver, but if the propagation geometry and motion are predominantly horizontal, all scaling factors will be similar and compensating for the average Doppler usually suffices. Scattering by suspended particles may complicate the observed Doppler and multipath profiles, and is beyond the scope of this work.

Given an estimate of $\beta$ the Doppler-compensated received signal is obtained from $y_{m}(t)$ as

$$
y_{m}^{\prime}(t)=y_{m}\left(\frac{t}{1+\beta}\right) e^{-j \omega_{c}[\beta t /(1+\beta)]},
$$

and used in all subsequent TR processing. The correctness of Eq. (9) can be asserted for the Doppler-distorted complex envelope of Eq. (7), $y_{m}(t)=x((1+\beta) t) e^{j \omega_{c} \beta t}$, in which case $y_{m}^{\prime}(t)=x(t)$ as intended. The same Doppler correction is applied to received channel probes whenever they are available. This operation, which is shown to preserve the sharpness of the QF in Sec. A 1, leads to equivalent pulse shapes and impulse responses that are much more convenient to process and visualize due to their comparatively low rate of variation in time. A similar resampling approach has been proposed by Song et al., ${ }^{11}$ but justified only heuristically. Alternative Doppler compensation methods are needed when $\beta$ cannot be assumed constant over a packet duration, but this hypothesis proved to be fully satisfactory for MREA'04 data.

The type of Doppler processing proposed here bears some resemblance to methods that have been proposed for varying the focal range of an active TRM through frequency shifting of a single captured probe. ${ }^{21,22}$ The approach relies on the fact that, for a given environment, nominal frequency $\omega$ and range $r$, the ratio between (small) relative changes $\Delta \omega / \omega$ and $\Delta r / r$ is a constant known as the waveguide invariant. In a broadband communications context this property implies that the channel impulse response between a static source and a receiver at range $\Delta r+r$ approximately equals a time-scaled version of the nominal one. ${ }^{23}$ The Doppler processing method described above can then be interpreted as follows: The packet transmission originating at range $r$ is time scaled at the TRM to compensate for Doppler compression due to source motion, but this also rescales the underlying impulse response and makes it appear as though the source is positioned at a different range. Direct matched filtering using measured probes would then result in loss of sharpness of the QF due to range mismatch. Time scaling of channel probes prior to filtering counters this effect by replicating exactly the same impulse response distortion introduced in data packets, so that the range mismatch vanishes and a sharply focused signal is again obtained.

\section{MULTICHANNEL COMBINING}

A sum of matched filters such as the one used in PPC is a known generic front end for optimal multichannel data receivers under several criteria, including minimum MSE (Ref. 24) and minimum probability of sequence error under additive white Gaussian noise. ${ }^{25}$ It should ideally be followed by a single-channel receiver to deal with residual ISI.
Alternative strategies for multichannel equalization have been proposed for reducing the overall computational complexity or providing more flexibility when the channel responses are imperfectly known at the receiver. ${ }^{1}$

Formal justification for the multipath compensation property can be found elsewhere, ${ }^{1,2,26}$ but intuitively it may be understood as follows. Each term $h_{m}^{*}(-t) * h_{m}(t)$ in Eq. (3) has a main lobe at time $t=0$ and (conjugate symmetrical) secondary lobes at other delays due to multipath. Main lobe contributions are all real, positive, and hence add up in phase. Secondary lobes, however, are not aligned in delay and phase for different sensors, and are not expected to reinforce each other the way that the main lobes do. As a result, an impulselike QF with dominating main lobe emerges as more and more terms are added. Perfect ISI removal through time reversal is only theoretically attained in the limit as the number of individual multipaths and/or uncorrelated receive elements gets very large.

The proposed multichannel combining algorithms are based on the assumption that, for moderate mismatch, the constructive interference of pulse contributions in $q(0)$ is partially lost even though the shapes of individual terms in the summation are not severely affected with respect to the static case. It should then be possible to mitigate this effect by multiplying each term by a single complex coefficient $w_{m}$ to restore the phase alignment at $t=0$. Denoting by $h_{m}^{\prime}(t)$ the actual pulse shape during focusing that differs from the channel probe $h_{m}(t)$, the modified TRM output in the presence of mismatch is given by

$$
\begin{aligned}
& z(t)=\sum_{m=1}^{M} w_{m} z_{m}(t)=\sum_{k} a(k) q\left(t-k T_{b}\right), \\
& q(t)=\sum_{m=1}^{M} w_{m} q_{m}(t), \quad q_{m}(t)=h_{m}^{*}(-t) * h_{m}^{\prime}(t) .
\end{aligned}
$$

In terms of symbol-rate-sampled variables in Eqs. (10) and $(11), z(n) \triangleq z\left(n T_{b}\right), z_{m}(n), q(n), q_{m}(n)$, one seeks to choose the coefficients $w_{m}$ so that $q(n)$ approximates a discrete impulse and hence $z(n) \propto a(n)$. Note that the same notation is used for matched and mismatched $q$-functions in Eqs. (4) and (11); unless otherwise stated (e.g., in DDPPC), the mismatched case (11) will be assumed henceforth.

\section{A. Numerical simulation}

The main goal of this section is to provide motivation for the practical multichannel combining cost functions to be presented in Sec. III B by examining several approaches for merging QF contributions. To this end, the impact of Eq. (10) on TR focusing is illustrated in a simulated scenario that resembles the conditions of the MREA'04 sea trial described in Sec. IV A. Note, however, that this is an idealized simulation with no noise and in which a clairvoyant receiver precisely knows the individual contributions $q_{m}(t)$ to the overall $\mathrm{QF}$ as defined in Eq. (11).

The simulated environment is a range-independent ocean cross section with $130 \mathrm{~m}$ depth. The sound-speed profile, which was chosen as representative of MREA'04 mea- 


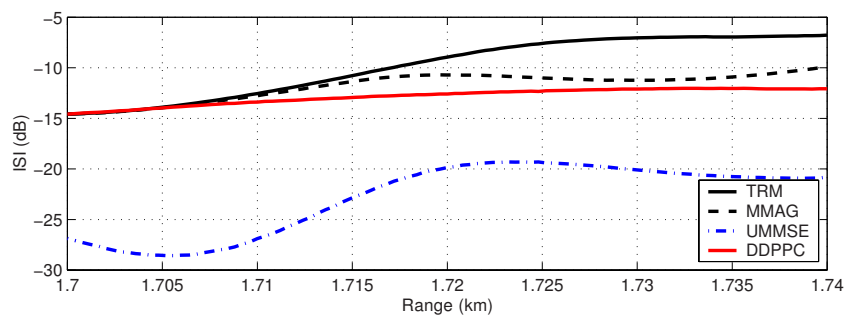

FIG. 1. (Color online) Evolution of the ISI metric, quantifying the similarity between the symbol-rate-sampled QF and a discrete impulse, in a simulated environment resembling the conditions of the MREA'04 sea trial (see Fig. 2). Results are shown for plain TRM, DDPPC, and two simplified criteria for multichannel combining (MMAG and UMMSE).

surements, is downward refracting with a thermocline at a depth of $20 \mathrm{~m}$. The source is located at $70 \mathrm{~m}$ depth and $1.7 \mathrm{~km}$ nominal range. Surface reflection was modeled as a deterministic angle-dependent coefficient equal to the average (coherent) specular component $\alpha_{S}=e^{-2(k \sigma \sin \theta)^{2}}$, where $k$ is the wave number at the carrier frequency of $3.6 \mathrm{kHz}, \sigma$ $=0.4 \mathrm{~m}$ is the root mean-square surface roughness, and $\theta$ is the grazing angle. A constant bottom reflection coefficient $\alpha_{B}=0.6$ was used. Attenuation/delay arrival data were generated with the Bellhop Gaussian beam ray tracer, ${ }^{27}$ and used to compute received pulse shapes (200 baud, $100 \%$ rolloff) across the eight array sensors, where the maximum delay spread is about $30 \mathrm{~ms}$. Delays were normalized so that the first arrival at the array always occurs at time 0 regardless of range.

Residual ISI. The source range was varied between 1.7 (nominal) and $1.74 \mathrm{~km}$, the mismatched QFs calculated according to Eq. (11) and sampled at symbol rate. Residual ISI at each range is quantified by ${ }^{28}$

$$
\operatorname{ISI}(q)=\frac{\sum_{n \neq 0}|q(n)|^{2}}{|q(0)|^{2}}=\sum_{n}\left|\frac{q(n)}{q(0)}-\delta(n)\right|^{2},
$$

which measures the similarity of $q(n)$, normalized to have unit magnitude at $n=0$, to an ideal discrete impulse $\delta(n)$. When $q(0)=1$ this coincides with the MSE $E\left\{|a(n)-z(n)|^{2}\right\}$ assuming that there is no noise and the symbols $a(n)$ are uncorrelated with unit power (Sec. II). Figure 1 depicts the behavior of the ISI metric for the following choices of combining coefficients $w_{m}$ in Eq. (11):

(1) Plain time reversal using $w_{m}=1$.

(2) Fully constructive interference of QF contributions at time 0 using $w_{m}=e^{-j \arg q_{m}(0)}$. As the maximum magnitude (MMAG) criterion of Sec. III B is an approximation of this, the same acronym is used in Fig. 1.

(3) The $q_{m}(n)$ are regarded as vectors over a finite time span around $0, \mathbf{q}_{m}=\left[q_{m}(n)\right]_{n=-D}^{D}$ and linearly combined by the $w_{m}$ to yield the best approximation to a discrete impulse $\boldsymbol{\delta}=[\delta(n)]_{n=-D}^{D}$ in the least-squares sense

$$
\arg \min _{w_{m}}\left\|\boldsymbol{\delta}-\sum_{m=1}^{M} w_{m} \mathbf{q}_{m}\right\|^{2} .
$$

This is approximated by the unconstrained minimum MSE (UMMSE) criterion of Sec. III B.
(4) DDPPC (Ref. 17) using $w_{m}=1$ but fully updated channel estimates at each range, as in Eq. (4).

Figure 1 shows that restoring the constructive interference in QF contributions (MMAG) can indeed improve the performance of TRM under moderate mismatch, although there is clearly more residual ISI than with full channel tracking (DDPPC). Unlike the other algorithms in Fig. 1, UMMSE directly optimizes a MSE criterion that is related to the ISI metric, which partially explains its large performance benefit for the nominal range. In fact, mismatched QF contributions under these idealized conditions are such that very accurate approximations to $\delta(n)$ can be found for other ranges as well, and interestingly these do not seem to involve fully constructive interference at time 0 . Note that the improvements afforded by MMAG, UMMSE, and DDPPC relative to plain TRM may vary significantly for other ranges and simulation setups.

Arguably, Fig. 1 should be interpreted with caution because UMMSE involves a form of channel inversion rather than pure channel matching, and therefore it is unfair to compare it to the other methods. Only in Sec. III B will practical approximate methods based only on TRM outputs be introduced, whose performance may legitimally be compared under a common MSE metric. Although channel inversion methods may potentially lead to smaller dispersion at the TRM output under ideal conditions, these are known to be much more sensitive to slight variations in the channel characteristics. This and the impact of noise help to explain the fact that performance gains of UMMSE using real data are much more modest than predicted here. In particular, the experimental results of Sec. IV B show that plain constructive interference of matched QF contributions using DDPPC provides lower ISI metrics.

\section{B. Cost functions}

Practical MC algorithms are formulated in terms of branch output sequences $z_{m}(n)$, rather than the underlying correlations $q_{m}(n)$ used in Sec. III A. A number of blind and nonblind (reference-driven) algorithms were developed in the course of this work, ${ }^{29}$ two of which are examined here. Regardless of design criteria, the primary metric for performance assessment in Sec. IV is MSE defined as $E\{\mid a(n)$ $\left.-\left.z(n)\right|^{2}\right\}$, where $a(n)$ denotes a transmitted symbol and $z(n)$ is the corresponding soft output of the receiver.

\section{Maximum magnitude}

Each coefficient in Eq. (10) performs a pure phase rotation, $w_{m}=e^{-j \theta_{m}}$, and the phases $\theta_{m}$ are chosen to maximize the expected squared magnitude of the mirror output. The first sensor is arbitrarily chosen as a reference by setting $w_{1}=1$. The cost function is

$$
J_{\mathrm{mag}}=E\left\{\left|z_{1}(n)+\sum_{m=2}^{M} z_{m}(n) e^{-j \theta_{m}}\right|^{2}\right\} .
$$

From $z_{m}(n)=q_{m}(n) * a(n)$ and the assumption of unit-power uncorrelated transmitted symbols introduced in Sec. II, Eq. (14) yields

Gomes et al:: Adaptive combining for time-reversed communications 


$$
J_{\text {mag }}=\sum_{n}\left|q_{1}(n)+\sum_{m=2}^{M} q_{m}(n) e^{-j \theta_{m}}\right|^{2} .
$$

If the system operates with low mismatch, such that $q(n)$ $\approx C \delta(n)$ in Eq. (11), then Eq. (15) will be largely dominated by the contribution for $n=0$. Ignoring the remaining terms, an optimal solution for $\theta_{m}$ is then readily given by $\theta_{m}$ $=\arg q_{m}(0)-\arg q_{1}(0)$.

In Appendix B a simple adaptation rule for the angles based on gradient ascent is derived by differentiating Eq. (14) with respect to $\theta_{m}$, obtaining a stochastic approximation to the gradient, and then using it as an error signal driving a phase-locked loop (PLL)-type filter. ${ }^{4}$ This yields

$$
\begin{aligned}
& \theta_{m}(n+1)=\theta_{m}(n)+K \Phi_{m}(n), \\
& \Phi_{m}(n)=\operatorname{Im}\left\{\left(z(n)-z_{m}(n) e^{-j \theta_{m}}\right)^{*} z_{m}(n) e^{-j \theta_{m}}\right\},
\end{aligned}
$$

where the loop gain $K$ is adjusted empirically. This adaptation rule does not require a reference signal and, as in most blind filtering algorithms, a residual phase ambiguity exists that manifests itself as a rotation of the signal constellation. Similar to plain TR, postprocessing is therefore needed to properly align and scale the output constellation.

\section{Unconstrained minimum MSE}

Rather than aligning the $z_{m}$ with unit-magnitude rotations, arbitrary coefficients $w_{m}$ can be used to minimize the output error. In this work an exponentially weighted leastsquares cost function is used,

$$
J_{\text {umse }}(n)=\sum_{k=0}^{n} \lambda^{n-k}\left|a(k)-\sum_{m=1}^{M} w_{m}(n) z_{m}(k)\right|^{2},
$$

so that time adaptation of the $w_{m}$ is actually carried out by the RLS algorithm (Ref. 30). Such a system effectively constitutes a very simple multichannel equalizer with one tap per sensor, which exploits probe preprocessing to significantly reduce the number of parameters to track. This approach will be termed UMMSE as in Ref. 29 although, strictly speaking, Eq. (18) is not a statistical criterion but rather a deterministic one. In reference-driven filtering schemes the packet symbols $a(k)$ to be used in Eq. (18) are assumed known during an initial training period, and afterward decisions based on the receiver output are used (decision-directed mode). Not only does this method handle phase synchronization, it also eliminates the need for output normalization.

\section{TRM postprocessing}

Symbol synchronization. In a practical TRM the output should undergo symbol synchronization to determine the time offset that maximizes a performance metric such as detection signal to noise ratio (SNR). Because the Doppler compensation technique of Sec. II A virtually eliminates any discrepancies in symbol rate, we simply calculate the $L$ polyphase components of the oversampled discrete-time output, $z^{(l)}(n) \triangleq z\left((l+n L) T_{b} / L\right), l=0, \ldots, L-1$, and choose the one with strongest average power. This is unnecessary when probes are estimated from data packets, as the best time off- set is known to be zero beforehand because channel identification removes delay ambiguities in pulse shapes.

Phase synchronization. Doppler compensation was found to be effective at eliminating carrier frequency mismatches that result in sustained rotation of the TRM output over time. Still, a popular PLL approach ${ }^{4}$ for phase synchronization was used as a postprocessor to track slow phase variations and hence properly align the output constellation. Specifically, a loop filter similar to Eq. (16) is used to update the estimated phase $\theta$, driven by the error signal $\Phi(n)$ $=\operatorname{Im}\left\{a(n)^{*} z(n) e^{-j \theta}\right\}$. Similar to Eq. (18), local symbol decisions should be used for computing $\Phi(n)$ upon entering decision-directed mode after the packet preamble. To simplify the comparison between different algorithms for ISI compensation (equalization, TRM, and multichannel combining), the same reference-driven phase synchronization method is used throughout this work. More appropriate choices are available for carrier synchronization in practical systems when ISI mitigation does not rely on an external reference. $^{18}$

Output normalization. The final operation to be performed after symbol synchronization and constellation alignment is to account for an unknown scaling introduced by the channel and amplifiers at the transmitter and TRM. This gain varies throughout data packets as the channel and QF change, and should therefore be tracked by an automatic gain control (AGC)-like system. A simple possibility is to recursively compute an exponentially weighted average of the unnormalized TRM magnitude

$$
\kappa(n)=\mu \kappa(n-1)+(1-\mu)|z(n)|, \quad 0<\mu \leq 1,
$$

and for a unit-magnitude constellation generate the normalized output as $z^{\prime}(n)=z(n) \kappa^{-1}(n)$. Strictly speaking, normalization is not required to slice M-PSK constellations, but it is useful for estimating output MSE values.

\section{EXPERIMENTAL RESULTS}

\section{A. The MREA'04 experiment}

The Maritime Rapid Environmental Assessment (MREA'04) sea trial ${ }^{31}$ was conducted on the continental shelf off the west coast of Portugal in April 2004, in an area to the north of the Setúbal Canyon shown in Fig. 2. The weather was calm during the acoustic trials, with sea state between 1 and 2, low wind of less than 10-15 knot, generally from the North quadrant, and wave height less than $2 \mathrm{~m}$. Extensive ground truth measurements of environmental parameters were performed before, during, and after the trial. ${ }^{31}$

The acoustic source was suspended from the NRV Alliance at depths ranging from 60 to $70 \mathrm{~m}$, depending on vessel speed (up to about $1.6 \mathrm{~m} / \mathrm{s}$ ). The TR experiment started at a close range of $0.6 \mathrm{~km}$ to the south of the receiver array $\left(38.36^{\circ} \mathrm{N}, 9.00^{\circ} \mathrm{W}\right)$ and the source progressively opened range to the southeast, up to $2 \mathrm{~km}$, along an approximately range-independent path with $110 \mathrm{~m}$ water depth and a 1.5 -m-thick silt and gravel sediment layer over a hard uniform sub-bottom. From Julian time 100.375 onward the Al- 
(m)

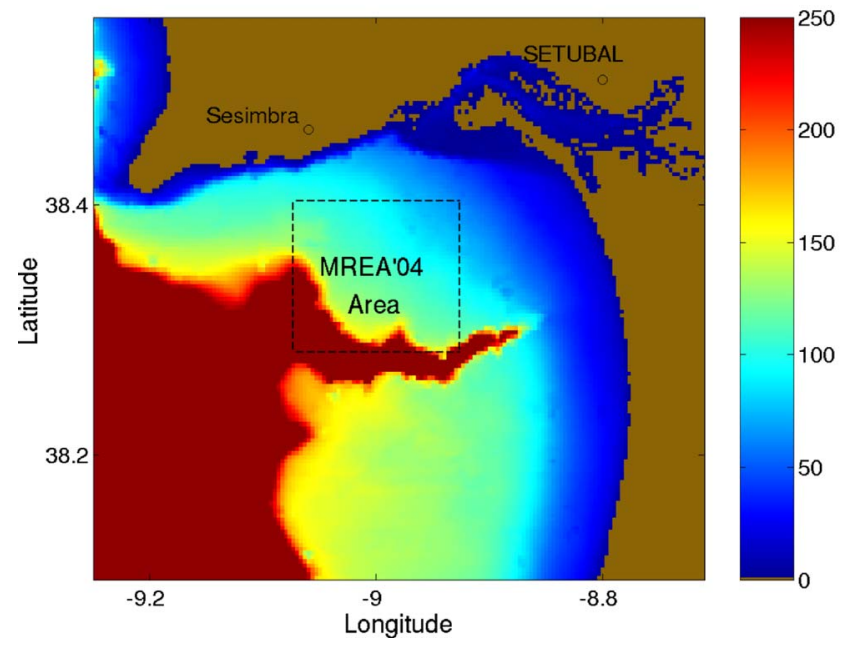

FIG. 2. (Color online) Site map for the MREA'04 sea trial, conducted off the west coast of Portugal in April 2004. The TR experiment took place in an approximately range-independent area $\left(38.36^{\circ} \mathrm{N}, 9.00^{\circ} \mathrm{W}\right)$ with $110 \mathrm{~m}$ depth and downward-refracting sound-speed profile. The drifting receiver array had eight hydrophones at depths $10,15,55,60,65,70,75$, and $80 \mathrm{~m}$. The acoustic source was suspended from the NRV Alliance at depths of $60-70 \mathrm{~m}$ and towed at up to $1.6 \mathrm{~m} / \mathrm{s}$. Throughout the experiment the source-array range varied from 0.6 to $2 \mathrm{~km}$.

liance maneuvered around a fixed position. The sound-speed profile was downward refracting, with a thermocline at a depth of about $20 \mathrm{~m}$.

The drifting receiver is an acoustic oceanographic buoy (AOB) developed at the University of Algarve. The AOB can digitize and record eight hydrophone signals in the frequency band $[0.1,16] \mathrm{kHz}$, sampled at up to $60 \mathrm{kHz}$. A wireless link (WLAN) provides remote access to status information and data snapshots at ranges up to $10-20 \mathrm{~km}$. In addition to eight hydrophones, vertically placed at depths $10,15,55,60,65$, 70,75 , and $80 \mathrm{~m}$, the AOB also has a 16 -sensor thermistor chain spanning $80 \mathrm{~m}$ for water column temperature monitoring.

During a period of approximately 90 min modulated data were transmitted at a carrier frequency of $3.6 \mathrm{kHz}$, using symbol rates of 200 or 400 baud, and both 2-PSK and 4-PSK constellations. As discussed in Sec. II, fourth-root raised-cosine signaling pulses with $100 \%$ rolloff were used to simplify out-of-band noise removal at the receiver by matched filtering to the transmitted pulse shape. The signal bandwidth is therefore $400 \mathrm{~Hz}$ at 200 baud and is $800 \mathrm{~Hz}$ at 400 baud. Each individual transmission comprises a single truncated signaling pulse (ping) acting as a channel probe with symmetrical guard intervals for a total duration of $1 \mathrm{~s}$, followed by a $20 \mathrm{~s}$ data packet. To enhance the SNR when directly measuring channel responses, probe pulses were sent with double the amplitude of signaling pulses in data packets. The source sequentially transmitted four packets for each of the following modulation formats: 2-PSK/ 200 baud, 2-PSK/ 400 baud, 4-PSK/ 200 baud, and 4-PSK/ 400 baud. The whole activity cycle, lasting for $336 \mathrm{~s}$, was repeated every $360 \mathrm{~s}$. The data set analyzed here comprises 200 probe/ packet pairs.

\section{B. Performance analysis}

Received signals were passband filtered, sampled at $20080 \mathrm{~Hz}$, and converted to baseband. Packets were classified and frame synchronized by crosscorrelation with the first 2 kilosamples of all known modulated wave forms, then match filtered by the appropriate fourth-root raised-cosine pulse and resampled (oversampled) at $L=4$ times the symbol rate. No attempt was made to detect the channel probes; they were segmented based on their known position relative to the beginning of data packets, then match filtered and resampled as above.

\section{Notes on filtering}

Throughout Sec. IV B $(m, n)$ will denote the length of a single-channel filter with $m$ causal coefficients and $n$ anticausal ones. A multiple-input/single-output filter comprising $p$ single-channel parallel filters of length $(m, n)$ whose outputs are added to create a scalar output will be denoted by $(m, n) \times p$. Such $p$-channel filters are used when processing fractionally sampled communications wave forms and/or multisensor data, such that $p$ equals the oversampling factor (relative to the symbol rate) times the number of sensors. In practical reference-driven filtering schemes, $n$ anticausal coefficients are effectively obtained when the reference signal is delayed by $n$ samples with respect to the received signals. In non-reference-driven (blind) schemes the distinction between causal and anticausal coefficients is meaningless and $(m, n)$ should simply be interpreted as a filter with $m+n$ coefficients.

Regarding equalization, choosing a good combination of filter lengths from channel estimates under fractional sampling is known to be unreliable and often done offline by trial and error. For decision-feedback equalizers (DFEs) popular design guidelines ${ }^{32}$ recommend using the feedback filter to cancel causal (postcursor) ISI, whereas feedforward filters will be much shorter to capture multipath energy and cancel anticausal (precursor) ISI. In this work appropriate equalizer lengths were set empirically for each packet in each experiment by searching over a plausible range of candidate lengths and selecting the one yielding the best performance. Somewhat unexpectedly, the best lengths reported below were found to be consistent across a clear majority of both 200 and 400 baud packets.

As in other references, ${ }^{33}$ the impact of symbol errors on the performance of reference-driven channel estimation, equalization, and phase tracking algorithms is not addressed in this work. These subsystems are always operated in training mode, where the correct symbols are known, and the calculated performance metrics should then be interpreted as optimistic estimates of what could actually be achieved. For values of output MSE higher than about $-5 \mathrm{~dB}$ the number of symbol errors is sufficiently large to have a significant impact on performance in decision-directed mode. This might cause divergence of adaptive algorithms if corrective measures are not implemented, such as freezing the updating of coefficients when unreliable decisions are detected. 


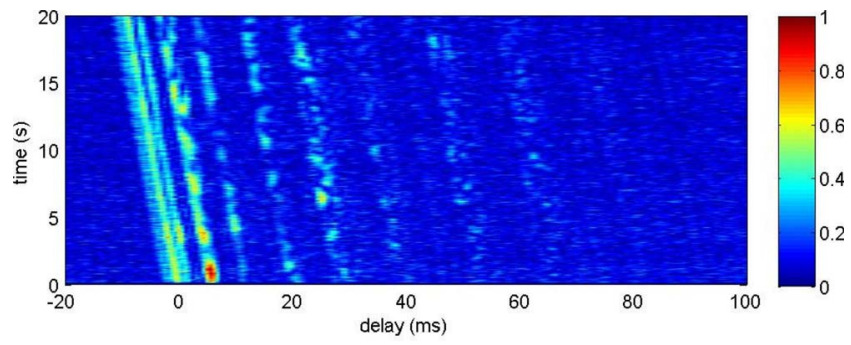

(a)

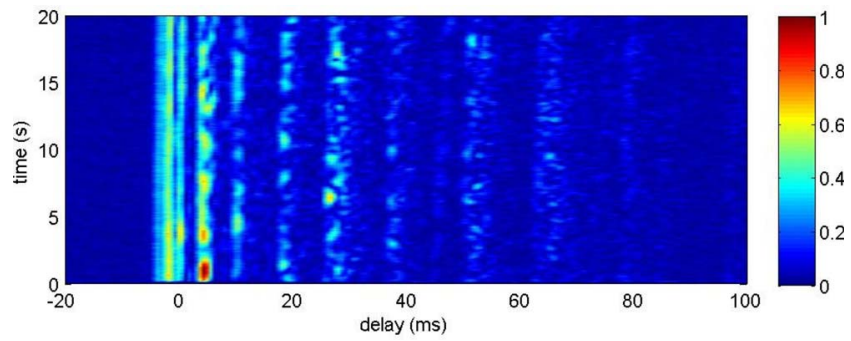

(b)

FIG. 3. (Color online) Evolution of amplitude-normalized estimated channel responses at depth $75 \mathrm{~m}$ (hydrophone 7) for packet 149 (400 baud). A horizontal slice through any of the plots represents a snapshot of the timevarying response. The coherence time for this channel was estimated to be about $1 \mathrm{~s}$. Estimates are based on RLS transversal filtering, fouroversampling, filter order $(41,10)$ per polyphase component (for order notation see Sec. IV B 1), and $\lambda=0.95$. (a) Before Doppler compensation, $f_{d}$ $=1.65 \mathrm{~Hz}$ at the carrier frequency of $3.6 \mathrm{kHz}$. (b) After broadband Doppler compensation as described in Sec. II A.

\section{Channel responses}

Figure 3(a) shows the evolution of the estimated impulse response at depth $75 \mathrm{~m}$ in one of the received 400 baud packets (PKT 149) with significant Doppler distortion. Channel identification was performed independently for each sensor using the exponentially windowed RLS algorithm. ${ }^{30}$ For computational efficiency, each $L$-oversampled hydrophone signal was split into $L$ polyphase components, $y_{m}^{(l)}(n)=y_{m}(l$ $+n L)$, and these were used as references to a bank of $L$ parallel RLS transversal filters fed by the known packet symbols. Each filter operates with 41 causal and 10 anticausal coefficients [abbreviated as $(41,10)$ using the notation introduced in Sec. IV B 1] and forgetting factor $\lambda=0.95$ empirically adjusted to minimize the residual error variance. This technique decreases the overall computational complexity by a factor of $L$ relative to direct identification of $y_{m}(n)$ from the zero-interpolated symbol sequence. Snapshots of the RLS coefficient vectors (estimated polyphase components of impulse responses) were taken every 20 symbol intervals and rearranged in the correct temporal order to produce the plot. The multipath arrival structure, spanning about $50 \mathrm{~ms}$, is reasonably sparse and clearly visible in Fig. 3(a), as well as a time compression due to Doppler that causes the arrivals to slip by 14 samples (3.5 symbols) in the course of a $20 \mathrm{~s}$ packet. Figure 3(b) shows the impulse response estimate for the same packet after Doppler compensation as described in Sec. II A and Appendix A, where the multipath structure is seen to remain essentially unaltered. The coherence time for the channel of Fig. 3(b) was estimated to be about 1 s (Doppler bandwidth of $1 \mathrm{~Hz}$ ). Once the average Doppler scaling

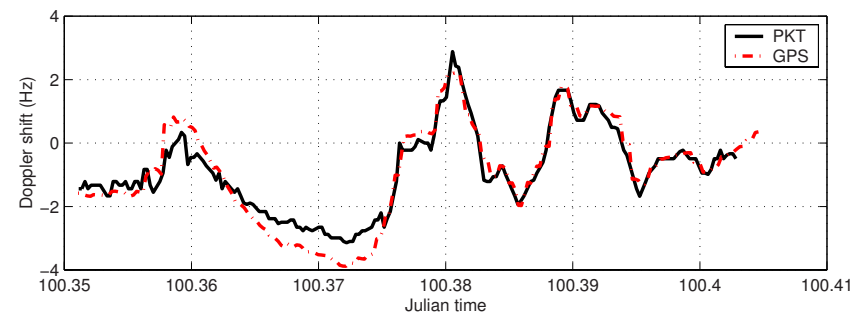

FIG. 4. (Color online) Estimated Doppler shift at the carrier frequency $f_{c}$ $=3.6 \mathrm{kHz}$ from packet data and GPS navigation data. GPS-based estimates were obtained by determining the source velocity along the source-receiver direction, calculating the time scaling factor $\beta$ according to Eq. (8), and plotting $f_{c} \beta$. In packet-based estimates $\beta$ was directly obtained from the ratio between received and transmitted packet durations, averaged across all receiver hydrophones.

in received signals was compensated, it was found that including other dedicated symbol synchronization subsystems at the receiver was unnecessary. The various structures described below use fractional sampling, which can automatically perform fine adjustments to the sampling instants when needed.

The causal/anticausal filter lengths used in Fig. 3 were empirically chosen to capture most of the multipath energy in all 400 baud packets of the data set. In 200 baud packets the filter lengths used for channel identification could be reduced to $(21,7)$ without significantly affecting the residual error, i.e., while still capturing all relevant multipaths.

Figure 4 shows estimates of Doppler shift at the carrier frequency, $f_{c}$, obtained from (i) measurements of packet time scaling $\left(f_{d}=f_{c} \beta\right.$, see Appendix A) and (ii) navigation data from the global positioning system (GPS) obtained at the source and receiver. The agreement between both curves is very good, suggesting that the physically motivated Doppler resampling procedure (9) is indeed plausible. As will be seen presently, best results are obtained in the low Doppler region to the right in Fig. 4, where the Alliance was relatively stationary.

Regarding input power levels, Fig. 5 shows the evolution of the signal to interference plus noise ratio (SINR) estimate defined in Eq. (C3) of Appendix C. It makes sense to

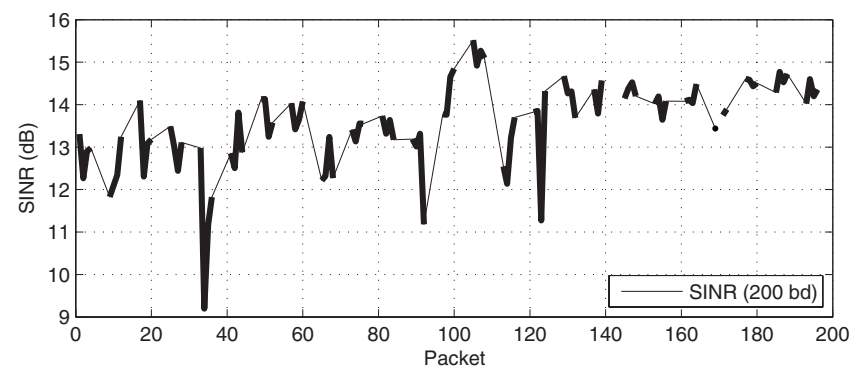

FIG. 5. Estimated input SINR (see Appendix C) based on channel identification [RLS transversal filtering, four-oversampling, order $(21,7)$ per polyphase component]. Forgetting factors were varied in the range 0.93 $\leqslant \lambda \leqslant 0.98$, and observed MSE values were fitted to theoretical expressions accounting for excess adaptation MSE in RLS to estimate the actual power of interferences (ambient noise and reverberation). Signal power is directly given by the norm of the RLS coefficient vector for the best forgetting factor. SINR estimates are averaged across all receiver hydrophones. Values for 400 baud packets are omitted, as the required filter lengths are outside the valid range of theoretical expressions for excess MSE in RLS. 


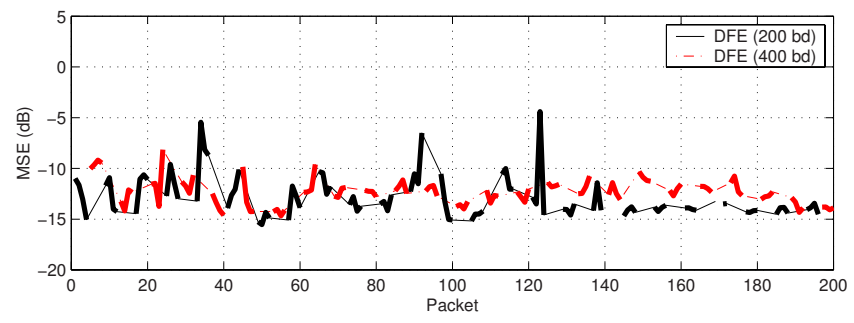

FIG. 6. (Color online) Performance of multichannel decision-feedback equalization using RLS, two-oversampling, eight sensors, $\lambda=0.95$. For each packet the lowest MSE obtained in a set of candidate equalizer lengths is shown. In most packets the best equalizer has $(2,1) \times 16$ feedforward and three feedback coefficients (for notation see Sec. IV B 1). The short feedback filter length suggests that the equalizer only exploits the arrivals shown in Fig. 3(b) up to $11 \mathrm{~ms}$.

adopt this metric as the definition of input $\mathrm{SNR}\left(\mathrm{SNR}_{\mathrm{in}}\right)$, as it attempts to account for the useful input signal energy that can be captured by RLS-based methods used for equalization and probe estimation, casting as interference the remaining received energy. To compute SINR as outlined in Appendix $\mathrm{C}$ the forgetting factor $\lambda$ was varied in the range $[0.93 ; 0.98]$. As in Fig. 3, lowest RLS residual errors were consistently obtained for $\lambda \approx 0.95$. The SINR estimates for 400 baud packets are not shown in Fig. 5, as the $(41,10)$ filter order that is needed for reasonable modeling of multipath is too large and leads to an invalid denominator in Eq. (C2).

Similar to SINR, a SNR measure at the output of a digital receiver is given by ${ }^{6,11,17} \mathrm{SNR}_{\text {out }} \approx \mathrm{MSE}^{-1}$ (see also Appendix C). For suitably defined SNR metrics, one expects to find $\mathrm{SNR}_{\text {out }} \leqslant \mathrm{SNR}_{\text {in }}$, where equality is attained when the receiver perfectly eliminates ISI without noise enhancement. ${ }^{6,11}$ As discussed below, optimal MSE values presented in Fig. 6 turn out to be in reasonably good agreement with SINR in Fig. 5, particularly for packets 120,..,200 where Doppler scaling is low and $\mathrm{MSE}^{-1} \approx \mathrm{SINR} \approx 14 \mathrm{~dB}$. This suggests that (i) the effective input SNR is well captured by SINR and (ii) equalization essentially achieves the practical lower bound for output MSE, so these results can be used to assess the performance degradation incurred by alternative TR-based receivers.

\section{Equalization}

To benchmark the performance of TRM demodulation algorithms, data packets were processed by a conventional RLS-based $\quad(\lambda=0.95) \quad$ multichannel decision-feedback equalizer. ${ }^{25}$ Given the effectiveness of Doppler compensation, the equalizer was able to cope with residual phase fluctuations without the need for a carrier recovery subsystem. Presumably, such a system would be required with higher carrier frequencies. The full set of eight sensor wave forms was used, fractionally sampled by $L=2$. Fractional sampling of band limited single-carrier amplitude/phase-modulated signals eliminates aliasing and avoids having to estimate and track a single optimal sampling instant per symbol interval, using a timing recovery loop, ${ }^{18}$ prior to equalization. The technique is used throughout this work either for stand-alone equalization or cascaded time reversal/equalization.

Figure 6 shows the best MSE values that were obtained on each packet by cycling over a set of candidate equalizer lengths. Most of these correspond to $(2,1)$ feedforward coefficients per polyphase component in each sensor [abbreviated as $(2,1) \times 16$ using the notation introduced in Sec. IV B 1] and three feedback coefficients. The equalizer time span is considerably shorter than the ISI duration shown in Fig. 3, but it agrees with the empirical determination of optimal TRM probe lengths reported in Sec. IV B 4. According to the DFE design guidelines outlined at the start of Sec. IV, the short feedback filter length suggests that the equalizer essentially exploits the arrivals shown in Fig. 3(b) up to the one at $11 \mathrm{~ms}$. This could be due to fluctuations in the remaining contributions that preclude the coherent combination of multipath energy. Distinct MSE curves are given for 200 and 400 baud packets, the former showing better performance in packets where low Doppler shifts indicate more stationary channel responses (e.g., packets $120, \ldots, 200$ ) because ISI spans fewer symbols. Under stronger Doppler (e.g., packets $40, \ldots, 80)$, the higher equalizer update rate in 400 baud packets seems to enable more effective tracking of channel variations and closes the MSE gap.

For completeness, the performance of multichannel fractionally spaced equalizers (FSE) was also evaluated. Results have been omitted here in the interest of space, but the MSE curves are similar to those of Fig. 6, shifted upward by about $0.5 \mathrm{~dB}$, and these are attained in most packets using $(2,1)$ $\times 16$ coefficients with the full set of eight sensors. Both the DFE and FSE use fractional sampling in the feedforward filter, but the former has an additional feedback filter that is fed by previous symbol decisions. If correct, these decisions enable the elimination of postcursor ISI without noise enhancement, whereas a FSE must compensate for both precursor and postcursor ISI using only the linear feedforward filter. Usually, this means that FSE filters will be longer than DFE feedforward filters, and the best output MSE will be higher due to noise enhancement.

The similarity of results between DFE and FSE (small MSE differences and similar optimal feedforward filter lengths) strengthens the previous argument that late arrivals in the MREA'04 data set lack coherence and hence cannot be effectively combined by these equalizers. The disparity between DFE and FSE will increase when equalization follows time reversal (see Fig. 8), as imperfect focusing results in longer-range, albeit mild, ISI.

As discussed in Sec. IV B 1, the above results assume that equalizers are only operated in training mode. However, comparable results are attained in most of the packets using a 400-symbol training sequence (which is used for probe estimation in TR-based algorithms below) followed by decision-directed adaptation if the RLS forgetting factor is increased to around $\lambda=0.99$ to achieve a larger effective averaging window.

\section{Plain time reversal}

Figure 7 shows the plain TRM output for a $200 \mathrm{baud} / 2$ PSK packet, postprocessed as described below regarding Fig. 8. Pulse shapes for time reversal were obtained by directly observing the response to the single pulse that precedes each packet (see Sec. IV A). The plot shows quite stable behavior of the real part of the TRM output, $\operatorname{Re}\{z(n)\}$, over the packet 


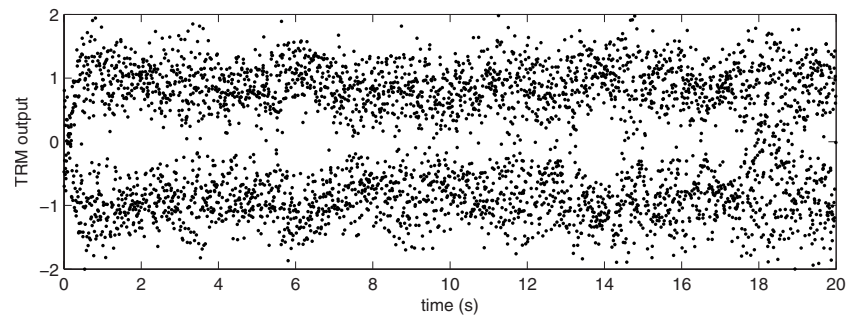

FIG. 7. Time evolution of the plain TRM output (real part) in PKT 155 (200 baud/2-PSK) using eight hydrophones. Pulse shapes for time reversal were obtained by directly observing the responses to the single pulse (ping) that precedes each packet. Postprocessing for symbol/phase synchronization and AGC is described in Sec. III C.

duration. TRM performance was found to be similarly stable in several other processed packets, which is somewhat unexpected given that the source was moving throughout much of the experiment, albeit at speeds not exceeding about $1.5 \mathrm{~m} / \mathrm{s}$. This contrasts with results reported by Flynn et al. ${ }^{17}$ where the collapse of plain TRM within 1 or $2 \mathrm{~s}$ of probe transmission motivated the development of DDPPC. The discrepancy may be attributed to the higher symbol rates (more than $2 \mathrm{kbaud}$ ) and denser multipath profile in that experiment, which probably translate into longer matched filters (probes) with less stable coefficients.

Figure 8(a) summarizes the MSE performance of plain TRM using observed probes (in short-term subintervals, see below). Results for 200 and 400 baud packets are not discriminated, as no significant differences in performance were found. Regarding the postprocessing steps of Sec. III C, phase alignment of the symbol-rate-synchronized TRM out-

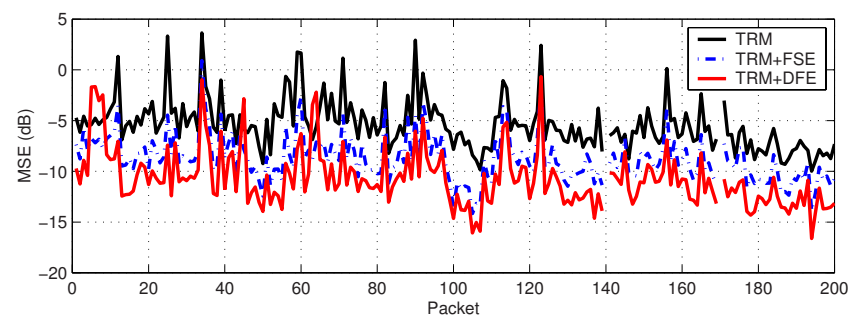

(a)

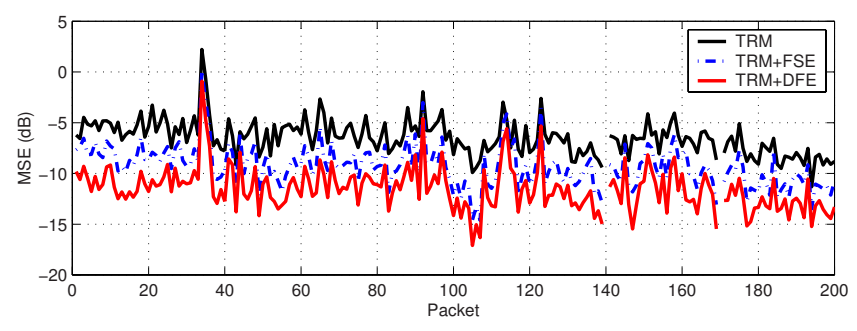

(b)

FIG. 8. (Color online) Performance of plain TRM using eight hydrophones and TRM with postequalization by DFE or FSE. MSE performance is evaluated on a short-term interval $0.5 \leqslant t \leqslant 2.5 \mathrm{~s}$. The equalizers use RLS, twooversampling, $\lambda=0.95$. For each packet the lowest MSE obtained in a set of candidate equalizer lengths is shown. In most packets the best DFE has $(3,4) \times 2$ feedforward and 20 feedback coefficients, whereas the best FSE uses mostly $(9,4) \times 2$ coefficients. (a) Observed probes from a single transmitted ping before each packet. (b) Estimated probes by channel identification on a 400-symbol packet preamble. RLS parameters for identification were set as described in Fig. 3. put is accomplished by a PLL whose generic loop filter update recursion (16) uses $K=5 \times 10^{-2}$. The AGC for output normalization (19) uses $\mu=0.995$, for an effective power averaging window length of about $(1-\mu)^{-1}=200$ samples. Whenever applicable, the same parameters are used in other TRM variants as well. When compared with the equalization results of Fig. 6 the MSE is seen to be higher by about $5 \mathrm{~dB}$, with stronger interpacket variations. Notice the reduction in MSE and improved consistency across packets in the lowDoppler region to the right of Fig. 8 (see also Fig. 4), which agrees with the equalization results of Fig. 6.

Figure 8(a) additionally shows MSE values when the TRM output, decimated to $L=2$ samples per symbol, is postprocessed by a DFE or a FSE. Within the set of considered equalizer lengths, best results for DFE were obtained with $(3,4) \times 2$ feedforward and 20 feedback coefficients. This short feedforward filter/long feedback filter combination is consistent with the DFE design guidelines described at the start of Sec. IV. Imperfect TR focusing using a sparse receiver array results in moderate, but longer-term, residual ISI (both precursor and postcursor), which explains the longer filter lengths needed to cope with it relative to plain multichannel equalization. For FSE $(9,4) \times 2$ coefficients yielded the best performance in most packets.

On average the MSE for TRM+DFE exceeds that of the multichannel DFE of Fig. 6 by $1.1 \mathrm{~dB}$. Figure 8(b) repeats the above results for probes estimated from the packet's initial 400 symbols, with identification parameters set as previously described in Sec. IV B 2. Lower MSE values are usually obtained with this method than with probe observation, and performance is more consistent across successive packets, but otherwise similar comments apply. Unless explicitly noted, probe estimation is used in the remainder of this section.

To quantify the degradation in TRM output over time, MSE values were averaged over short- $(S)$, medium- $(M)$ and long-term $(L)$ subintervals in each packet. These were defined as $S: 0.5 \leqslant t \leqslant 2.5 \mathrm{~s}, M: 4 \leqslant t \leqslant 6 \mathrm{~s}$, and $L: 10 \leqslant t$ $\leqslant 12 \mathrm{~s}$. The reference $t=0$ was set to the beginning of packets for observed probes and the end of the identification preamble for estimated probes. The following results were obtained.

Plain TRM. Figures 8(a) and 8(b) pertain to $S$ intervals. The average increases in MSE over $M$ and $L$ intervals relative to $S$ for all 200 baud (respectively, 400 baud) packets are 0.6 and $1.2 \mathrm{~dB}$ (respectively, 1.1 and $2.0 \mathrm{~dB}$ ). Increased sensitivity to mismatch at 400 baud was expected, as oversampled pulse shapes have finer temporal structure.

$T R M+D F E$. The average increases in MSE over $M$ and $L$ intervals relative to $S$ for 200 baud (respectively, 400 baud) packets are 0.2 and $0.4 \mathrm{~dB}$ (respectively, 1.1 and $1.7 \mathrm{~dB}$ ). Thus, while TRM+DFE provides significantly lower absolute MSE than plain TRM (Fig. 8), it was found that MSE fluctuations over time are not effectively attenuated by postequalization in 400 baud packets. This is consistent with Fig. 8, where the MSE of TRM+DFE tracks that of TRM at an approximately constant offset.

Actual MSE fluctuations from $S$ to $M$ or $L$ vary widely across packets, with standard deviations exceeding $1 \mathrm{~dB}$. 


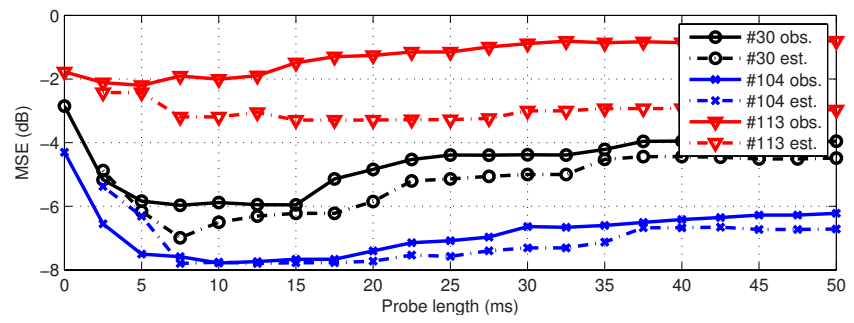

FIG. 9. (Color online) Impact of probe length on short-term plain TRM performance (eight hydrophones) for three individual packets (PKT 30, 104, 113) using direct probe measurements (obs.) or channel estimation from packet preambles (est.). Lowest MSE values are obtained for truncated probes of about 7-15 ms duration, which discard much of the multipath structure shown in Fig. 3. The trend for other packets (not shown) is similar, and in agreement with the short feedback filter lengths that were found for the equalization results shown in Fig. 6 .

Contrary to what was expected, no obvious correlation was found between the extent of MSE degradation in $M, L$ and the severity of Doppler distortion depicted in Fig. 4.

The above results were obtained with probe lengths optimized for each individual packet. Figure 9 depicts the evolution of short-term MSE in three representative packets as a function of probe length using either direct measurements (observed) or channel estimation from packet preambles (estimated). Interestingly, the lowest MSE values are obtained for truncated probes of about 7-15 ms duration, which discard much of the multipath structure shown in Fig. 3. This may be due either to the low energy of these discarded arrivals relative to the noise background or, more likely, to fluctuations that preclude the effective coherent combination of energy from those paths. Note that all reported MSE/ISI values for time reversal based on optimal probe lengths should be interpreted as lower performance bounds in practical systems where probes are truncated before focusing and packet decoding.

Finally, Fig. 10 shows the evolution of short-term MSE and bit error rate in TRM and TRM+DFE as a function of the number of sensors $M$, averaged over all packets. Array elements are sequentially selected from top, such that by increasing $M$ the size of the aperture increases as well. Reported experiments ${ }^{11}$ indicate that the performance of a long and dense TRM tends to saturate with growing $M$ after an initial rapid improvement, as the device already captures much of the energy in the water column and its ISI mitigation ability improves very slowly. By contrast, Fig. 10 shows a more linearlike trend (in decibel scale) as a function of $M$, with MSE gains dropping from $-1.5 \mathrm{~dB}$ for $M=2$ until $-0.7 \mathrm{~dB}$ for $M=8$. This suggests that the TRM still operates in a nonsaturated regime where each additional sensor introduces significant spatial diversity. Equivalently, impulse responses are sufficiently different between sensors spaced $5 \mathrm{~m}$ apart to ensure that QF contributions do not interfere constructively in the sidelobe region. The reduction in MSE by about $6 \mathrm{~dB}$ as $M$ varies between 1 and 8 is consistent with simulation results based on the parameters of Sec. III A. Postprocessing by the same DFE of Fig. 8 yields similar MSE improvements, in agreement with other TR experiments. ${ }^{11}$

The existence of a thermocline at $20 \mathrm{~m}$ induces rapid delay variations in the arrivals with low grazing angles as a function of depth, thus enhancing differences in the pattern of early arrivals (direct path and surface reflection) between the upper and lower sections of the array (located above and below the thermocline, respectively). When adding sensors to the TRM from top one would therefore expect to see

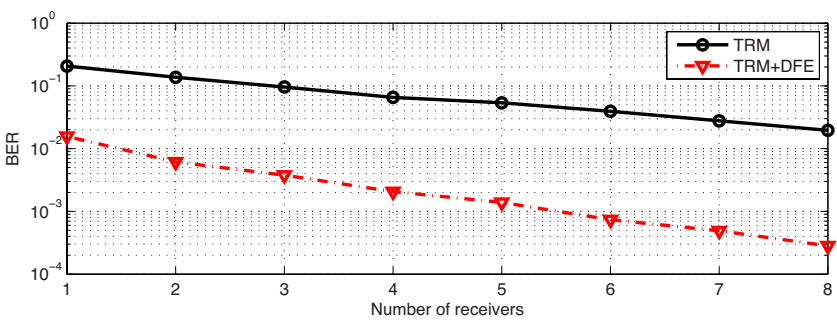

(b)

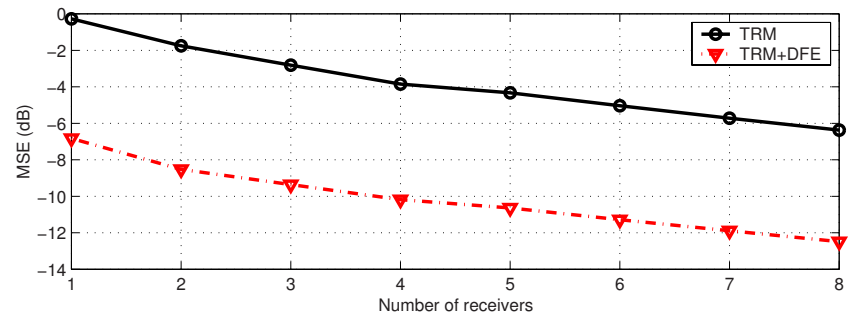

(a)

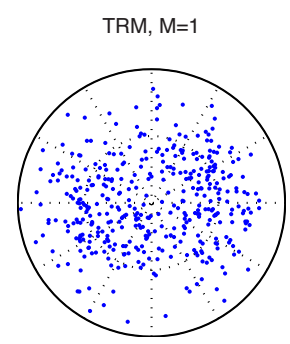

(c)
TRM, M=8

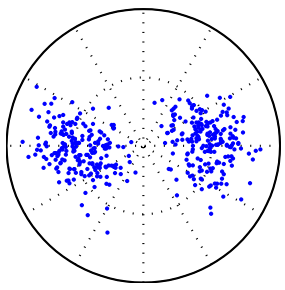

(d)
TRM+DFE, M=1

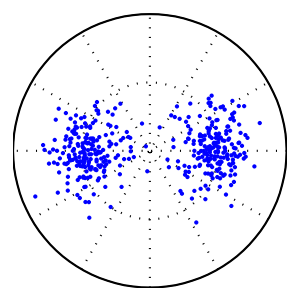

(e)
TRM+DFE, $M=8$

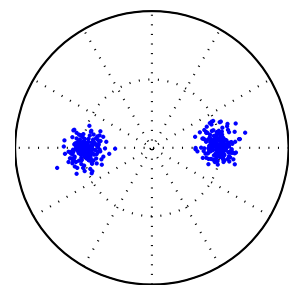

(f)

FIG. 10. (Color online) Impact of the number of sensors, $M$, selected from top, on the short-term performance of plain TRM and TRM with DFE postequalization [RLS, two-oversampling, (mostly) $(3,4) \times 2$ feedforward and 20 feedback coefficients, $\lambda=0.95]$. Probes estimated from packet preambles. (a) Output MSE, averaged over 200 packets. In this sparse TRM, saturation of MSE for sufficiently large $M$ is not yet visible. (b) Average bit error rate. [(c) and (d)] Scatter plots for PKT 155, plain TRM, $M=1$ and 8. [(e) and (f)] Scatter plots for TRM+DFE. 
lower performance gains once the thermocline is crossed, from $M=3$ onward, as received pulse shapes become more similar. Although not very clearly visible, the slope of the upper curve in Fig. 10(a) does decrease for $M \geqslant 4$. The fact that the inflection point is not located at $M=3$ could be due to the observed partial confinement of acoustic energy in the region below the thermocline, so that adding sensors for $M$ $\geqslant 3$ provides additional signal power that improves performance beyond what would be expected by considering delay disparities alone.

\section{Multichannel combining}

The performance gains of proposed MC algorithms (MMAG and UMMSE) and DDPPC were quantified by comparing their MSEs with that of plain TRM in $S, M$, and $L$ packet subintervals. The following results were obtained.

$M M A G$. The average improvements in MSE relative to plain TRM in the $S, M$, and $L$ intervals for 200 baud (respectively, 400 baud) packets are $0.3,0.5$, and $0.8 \mathrm{~dB}$ (respectively $0.3,0.5$, and $1.0 \mathrm{~dB}$ ). MSE gains increase as one progresses from $S$ to $M$, and $L$, indicating that MMAG can partially compensate for the degradation in performance of plain TRM due to mismatch. Improvements of less than $1 \mathrm{~dB}$ are modest, but nonetheless these represent $10 \%-20 \%$ of the average MSE of plain TRM in the $M$ and $L$ regions.

$U M M S E$. MSE gains over TRM in the $S, M$, and $L$ intervals for 200 baud (respectively, 400 baud) packets are 3.5, 4.0, and $4.3 \mathrm{~dB}$ (respectively 2.4, 2.9, and $3.5 \mathrm{~dB}$ ). UMMSE yields larger MSE gains than MMAG and DDPPC, which is not surprising as this is an equalization-based scheme that minimizes a MSE-like performance metric.

$D D P P C$. $S, M$, and $L$ MSE gains for 200 baud (respectively, 400 baud) packets are 1.0, 1.6, and $2.3 \mathrm{~dB}$ (respectively, $-0.5,0.5$, and $1.4 \mathrm{~dB}$ ). Channel tracking is seen to improve upon plain TRM under mismatch, but the MSE gains are actually smaller than those afforded by UMMSE and the number of parameters to track is substantially higher.

In MMAG $K=5 \times 10^{-2}$ is used in the loop filter update recursion (16) for each weight $w_{m}=e^{-j \theta_{m}}$. The same parameters for output phase synchronization and AGC used in plain TRM are also adopted in MMAG and DDPPC (the RLS-based UMMSE algorithm requires neither of them, and uses $\lambda=0.95$ ).

Similar to what was done for TRM and TRM+DFE in Sec. IV B 4, the self-degradation of the above algorithms was assessed by comparing their MSE in $M / L$ intervals with $S$.

MMAG. The average MSE in $M$ and $L$ in 200 baud (respectively, 400 baud) packets increases by 0.4 and $0.7 \mathrm{~dB}$ (respectively, 0.8 and $1.3 \mathrm{~dB}$ ) relative to $S$. The values are lower than those found for TRM, indicating that the algorithm is more robust to channel variations.

UMMSE. Similar comments apply to this algorithm, where MSE increases by 0.2 and $0.4 \mathrm{~dB}$ (200 baud) or 0.7 and $0.9 \mathrm{~dB}$ (400 baud).

$D D P P C$. Not surprisingly, DDPPC provides essentially constant performance throughout packets. The average degradations in MSE are -0.03 and $-0.2 \mathrm{~dB}$ (200 baud) or 0.1 and $0.03 \mathrm{~dB}$ (400 baud).
Although MMAG and UMMSE have been proposed as low-complexity alternatives to DDPPC, note that DDPPC and UMMSE (or MMAG, for that matter) can be readily combined into a composite algorithm that will attain the best overall performance.

To conclude the analysis, QFs and ISI metrics were computed as in Sec. III A and plotted in Figs. 11(a), 11(f), and 11(k). Actually, these show the difference in ISI metric relative to plain TRM in the $S, M$, and $L$ regions of individual packets, in line with what was done previously for MSE. Again, it can be concluded that these algorithms partially compensate for mismatch, yielding increasing gains over TRM as time progresses. This, too, can be seen in the scatter plots included in Fig. 11. Note also that, while UMMSE achieves lower MSE than DDPPC, its residual ISI is higher. This might be attributed to the nonequivalence of both performance metrics in the presence of noise (which was absent in the simulation reported in Sec. III A), in which case MMSE solutions are known to retain some residual ISI. ${ }^{18}$ The observed inversion in relative performance of UMMSE and DDPPC in terms of MSE and ISI could also be due to inaccuracies in the method used to estimate the QFs. In fact, probe shapes were first estimated throughout each packet as described previously, snapshots taken every 100 symbol intervals, QF contributions computed according to Eq. (11), and then linearly combined by a set of weighting coefficients that reflect the intended behavior of the various algorithms, as described in Sec. III A. In the case of MMAG and UMMSE, discrepancies with respect to the actual coefficients computed according to Sec. III B may exist.

\section{CONCLUSION}

Experimental results were presented demonstrating demodulation of 200/400 baud PSK data collected during the MREA'04 sea trial in an eight-sensor receiver array. Several receiver architectures were examined and compared, namely, multichannel equalization, passive TRM with and without postequalization, DDPPC, and two multichannel combining methods (MMAG and UMMSE). The analysis included issues such as the characterization of time variability in channel responses and the impact on TRM performance of probe length, probe observation/estimation, and the number of array sensors.

MREA'04 data were collected with a moving source and drifting receiver, resulting in Doppler scaling of wave forms that was compensated by a simple resampling method. The technique was found to be very effective, generating nearly time-invariant equivalent channels where TR focusing often lasts for the full packet duration (20 s) with moderate degradation due to mismatch that does not strongly depend on the original Doppler distortion. Possibly, focusing would have been less stable at higher data rates, as reported in other TR experiments. Best TRM performance was obtained for truncated probes that retain multipath energy only on a few arrivals, suggesting that the remaining ones were less stable and could not be coherently combined to enhance the signal energy at the mirror output. 


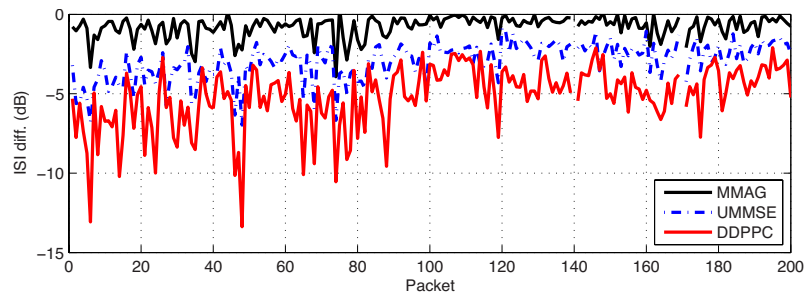

(a)

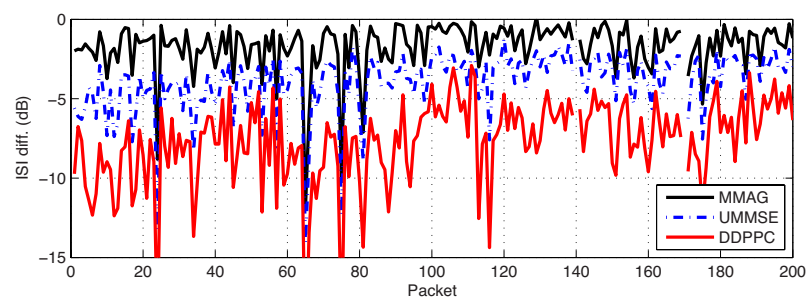

(f)

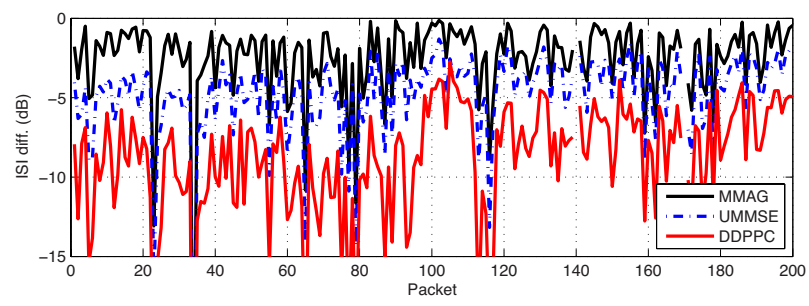

(k)

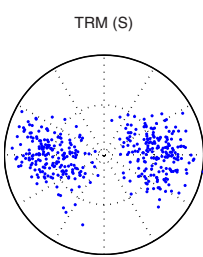

(b)

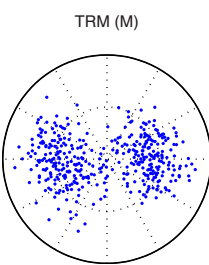

(g)

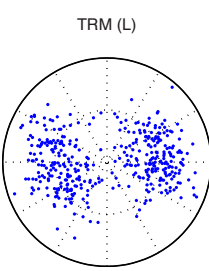

(1)

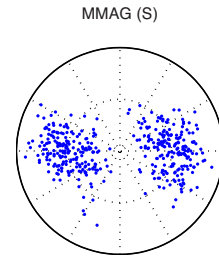

(c)

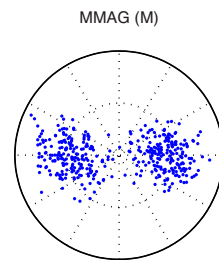

(h)

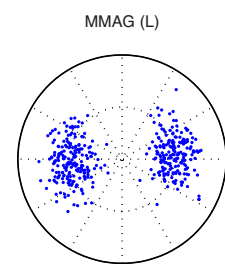

(m)

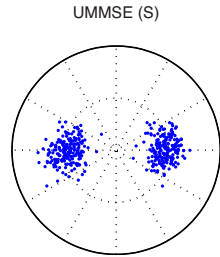

(d)

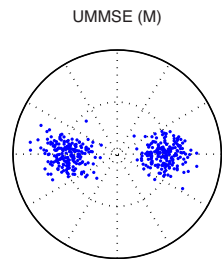

(i)

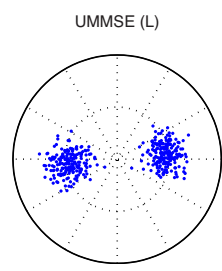

(n)

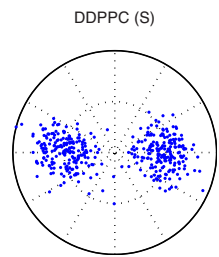

(e)

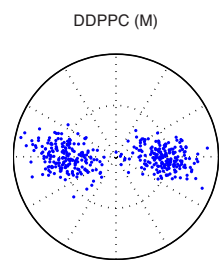

(j)

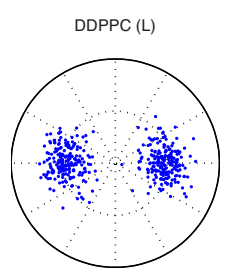

(o)

FIG. 11. (Color online) ISI reduction, on individual packets, of MC algorithms relative to plain TRM using eight hydrophones and estimated probes. Results are shown for MMAG, UMMSE, and DDPPC on short-term intervals $0.5 \leqslant t \leqslant 2.5 \mathrm{~s}$, medium-term intervals $4 \leqslant t \leqslant 6 \mathrm{~s}$, and long-term intervals $10 \leqslant t$ $\leqslant 12 \mathrm{~s}$. Larger ISI gains as $t$ increases indicate that the algorithms can compensate for some of the degradation in plain TRM due to environment mismatch. (a) Short-term ISI reduction. [(b)-(e)] Scatter plots for PKT 155 in short-term intervals for plain TRM, MMAG, UMMSE, and DDPPC. (f) Medium-term ISI reduction. [(g)-(j)] Scatter plots. (k) Long-term ISI reduction. [(1)-(o)] Scatter plots.

Plain TRM incurs a significant performance penalty relative to multichannel equalization, but postequalization was found to narrow the gap to about $1 \mathrm{~dB}$. The latter presents a lower-complexity alternative to multichannel equalization, but the computational savings were small in MREA'04 data, where very short multichannel filters achieved the best performance. Again, the conclusion might be different at higher data rates.

The MMAG and UMMSE algorithms were proposed to compensate for (moderately) degraded focusing due to mismatch by adaptively weighting, rather than simply adding, sensor contributions. Unlike DDPPC, they do not require tracking of received pulse shapes, which makes them less computationally intensive. Temporal stability results for the various algorithms showed that MMAG and UMMSE can indeed improve upon plain TRM, although the performance is not as stable as in DDPPC. The average MSE improvement of MMAG was modest, partly due to the good stability of plain TRM itself in many of the packets. UMMSE is actually a multichannel equalization approach with a single coefficient per sensor that outperformed DDPPC in terms of output MSE and provided improvements of 3-4 dB over TRM.

This work considered MMAG and UMMSE as alternatives to DDPPC, but these can actually be combined to create multichannel receivers with adjustable pulse shapes and sensor weighting coefficients. Developing adaptation criteria for such structures, accounting for the effect of channel uncertainties, and establishing links with channel-estimation-based equalization are topics for future research.

\section{ACKNOWLEDGMENTS}

This work was supported by Fundação para a Ciência e a Tecnologia (ISR/IST plurianual funding) through the PIDDAC Program and project NUACE POSI/CPS/47824/ 2002. The authors would like to thank the NATO Undersea Research Centre for the organization of the MREA'04 sea trial, NRV Alliance master and personnel, and the scientist in charge Emanuel F. Coelho. Several helpful comments and suggestions by the anonymous reviewers are also gratefully acknowledged.

\section{APPENDIX A: DOPPLER COMPENSATION}

Assume first that a single propagation path with impulse response $g_{m}(t)$ exists between the source and the $m$ th mirror sensor. Using Eq. (7), if the original baseband transmitted signal is denoted by $x(t)$, then from the receiver viewpoint the path is excited by $x((1+\beta) t) e^{j \omega_{c} \beta t}$ and the received signal is given by the convolution 


$$
\begin{aligned}
y_{m}(t) & =\int x((1+\beta)(t-\tau)) e^{j \omega_{c} \beta(t-\tau)} g_{m}(\tau) d \tau \\
& =\int x\left(t-\tau^{\prime}\right) \frac{e^{j\left[\omega_{c} \beta /(1+\beta)\right]\left(t-\tau^{\prime}\right)}}{1+\beta} g_{m}\left(\frac{\tau^{\prime}+\beta t}{1+\beta}\right) d \tau^{\prime} .
\end{aligned}
$$

This is recognized as a linear convolution between $x(t)$ and a time-varying impulse response whose magnitude equals $\left|g_{m}(\tau)\right|$ for $|\beta| \ll 1$, with the origin shifted to $-\beta t$. In other words, the shape of this impulse response is essentially obtained by gradually sliding $g_{m}(\tau)$ along the $\tau$ axis as $t$ progresses, such that the position of any feature in $g_{m}$ traces a line with slope $-\beta$ in the $(t, \tau)$ plane. This property can be shown to remain valid even for an impulsive response $g_{m}(t)=g_{m} \delta\left(t-\tau_{m}\right)$.

In a multipath channel $h_{m}(t, \tau)$ is the sum of individual path contributions $h_{m p}(t, \tau)$, each having a different Doppler shift $\beta_{p}$. The analysis of experimental data reveals that most often all the $\beta_{p}$ are approximately equal, and therefore all paths trace parallel trajectories (straight lines) in the $(t, \tau)$ plane [see Fig. 3(a)]. This happens when the propagation geometry and motion are predominantly horizontal, and suggests Doppler estimation algorithms based on identification of the time-variant impulse response and the common slope $\beta$ of multipath arrivals. While an offline method of this sort using the Radon transform was indeed used to compute the Doppler scaling in MREA'04 packets, it should be emphasized that the topic of Doppler estimation is not central to this work and other more practical options are available. For example, a simple method has been proposed whereby the compression is estimated by detecting and measuring the delay between known prolog and epilog sequences in each packet. $^{34}$

\section{Resampling}

Knowing the Doppler factor $\beta$ and its theoretical effect on the complex envelope of the transmitted signal (7), we compensate it according to Eq. (9) by canceling the term $e^{j \omega_{c} \beta t}$ and then resampling to eliminate the time scaling. Resampling in discrete time can be performed in a number of ways, e.g., using low-complexity parabolic interpolation. In this work an efficient polyphase implementation (MATLAB resample function) was used for block resampling of full packets.

Naturally, the question arises as to whether Doppler compensation disrupts TR focusing by disturbing the medium transfer function $g_{m}(t)$. To address that issue we proceed as previously, expressing the resampled signal as a convolution between the ideal transmitted signal $x(t)$ and a timevarying impulse response. Using Eqs. (9) and (A1) and performing a change of variables yields

$$
\begin{aligned}
y_{m}^{\prime}(t) & =y_{m}\left(\frac{t}{1+\beta}\right) e^{-j \omega_{c}[\beta t /(1+\beta)]} \\
& =\int x\left(t-\tau^{\prime}\right) \frac{g_{m}\left(\frac{\tau^{\prime}}{1+\beta}\right)}{1+\beta} e^{-j \omega_{c}\left[\beta \tau^{\prime} /(1+\beta)\right]} d \tau^{\prime} .
\end{aligned}
$$

This is a convolution between $x(t)$ and a time-invariant impulse response that may be easily related to the original medium response in the time and frequency domains

$$
\begin{aligned}
& g_{m}^{\prime}(t)=\frac{g_{m}\left(\frac{t}{1+\beta}\right)}{1+\beta} e^{-j \omega_{c}[\beta t /(1+\beta)]}, \\
& G_{m}^{\prime}(\omega)=G_{m}\left((1+\beta)\left(\omega+\frac{\omega_{c} \beta}{1+\beta}\right)\right) .
\end{aligned}
$$

The spectrum (A4) is a frequency-shifted and (slightly) rescaled version of the original $G_{m}(\omega)$, so it seems reasonable to expect that focusing will be preserved. Because both the packet and probe undergo the same Doppler compensation procedure, the residual ISI is determined by the new medium autocorrelation function which, similar to Eq. (5), is given by

$$
\begin{aligned}
\gamma^{\prime}(t) & =\sum_{m=1}^{M} g_{m}^{\prime *}(-t) * g_{m}^{\prime}(t) \\
& =\sum_{m} \int g_{m}^{\prime *}(\tau-t) g_{m}^{\prime}(\tau) d \tau \\
& =\frac{\gamma\left(\frac{t}{1+\beta}\right)}{1+\beta} e^{-j \omega_{c}[\beta t /(1+\beta)]} .
\end{aligned}
$$

As in Eq. (A4), the Fourier transform of this function is a frequency-shifted and rescaled version of the original one,

$$
\Gamma^{\prime}(\omega)=\Gamma\left((1+\beta)\left(\omega+\frac{\omega_{c} \beta}{1+\beta}\right)\right) .
$$

The (static) multipath compensation property of time reversal implies that $R(\omega) \Gamma(\omega) \propto R(\omega)$ in Eq. (5), so $\Gamma(\omega)$ is approximately flat in the signal band. In the MREA'04 experiment this band is at most $2 r_{b \max }=800 \mathrm{~Hz}$ for $r_{b}=400$ baud packets with $100 \%$ pulse rolloff. According to Eq. (8) $\beta_{\max }$ is about $10^{-3}$ for $v_{\max } \approx 1.5 \mathrm{~m} / \mathrm{s}$ and $c \approx 1.5 \times 10^{3} \mathrm{~m} / \mathrm{s}$, hence the maximum Doppler shift is about $f_{d \max }=\omega_{c} \beta_{\max } / 2 \pi$ $<4 \mathrm{~Hz}$ at the carrier frequency. Over the frequency band of interest $\left[\left(-r_{b \max } ; r_{b \max }\right)=(-400 ; 400) \mathrm{Hz}\right.$ in baseband $]$ the behavior of $\Gamma^{\prime}(\omega)$ is defined by the values of the original $\Gamma(\omega)$ in the interval $\left(-r_{b \max }\left(1-\beta_{\max }\right)-f_{d \text { max }} ; r_{b \max }(1\right.$ $\left.\left.+\beta_{\max }\right)+f_{d \max }\right)=(-403.2 ; 404.0) \mathrm{Hz}$. It may be concluded that if $\Gamma(\omega)$ is flat in the band of $R(\omega)$, then the same will be true for $\Gamma^{\prime}(\omega)$, with the possible exception of very narrow intervals at either the upper or lower edges of the signal band. With high probability time-reversed focusing will therefore be preserved by the proposed resampling method for Doppler compensation. 


\section{APPENDIX B: MMAG ADAPTATION RULE}

The cost function (14) is to be iteratively maximized over the set of real angles $\theta_{m}, m=1, \ldots, M$. Actually, only the gradient of Eq. (14) is needed to obtain an ascent iteration. To streamline the notation, the explicit dependence on the time instant $n$ of the various sequences appearing in this section will be dropped. The gradient $\partial J_{\text {mag }} / \partial \theta_{i}$ is calculated by lumping together all terms that are independent of $\theta_{i}$, viz.,

$$
\begin{aligned}
J_{\text {mag }} & =E\left\{\mid a_{i}-z_{i} e^{-j \theta_{i} \mid}\right\} \\
& =\sigma_{a_{i}}^{2}+\sigma_{z_{i}}^{2}-2 \operatorname{Re}\left\{x_{i} e^{-j \theta_{i}}\right\} \\
& =\sigma_{a_{i}}^{2}+\sigma_{z_{i}}^{2}-2 \operatorname{Re}\left\{x_{i}\right\} \cos \theta_{i}-2 \operatorname{Im}\left\{x_{i}\right\} \sin \theta_{i},
\end{aligned}
$$

where

$$
\begin{aligned}
& a_{i}=-z_{1}-\sum_{m \neq i} z_{m} e^{-j \theta_{m}}=-z+z_{i} e^{-j \theta_{i}}, \\
& x_{i}=E\left\{a_{i}^{*} z_{i}\right\}, \quad \sigma_{a_{i}}^{2}=E\left\{\left|a_{i}\right|^{2}\right\}, \quad \sigma_{z_{i}}^{2}=E\left\{\left|z_{i}\right|^{2}\right\} .
\end{aligned}
$$

The gradient with respect to $\theta_{i}$ is now readily obtained as

$$
\begin{aligned}
\frac{\partial J_{\mathrm{mag}}}{\partial \theta_{i}} & =2 \operatorname{Re}\left\{x_{i}\right\} \sin \theta_{i}-2 \operatorname{Im}\left\{x_{i}\right\} \cos \theta_{i} \\
& =2 \operatorname{Im}\left\{E\left\{\left(z-z_{i} e^{-j \theta_{i}}\right)^{*} z_{i} e^{-j \theta_{i}}\right\} .\right.
\end{aligned}
$$

A simple gradient ascent iteration is given by

$$
\theta_{i}(n+1)=\theta_{i}(n)+K \frac{\hat{\partial J}_{\mathrm{mag}}}{\partial \theta_{i}},
$$

where a common stochastic approximation to the gradient is used, which simply amounts to ignoring the statistical expectation in Eq. (B4). The stochastic gradient in Eq. (B5) may be viewed as an error signal driving a simple PLL, whose loop filter may be refined to obtain more robust tracking behavior. ${ }^{4}$ In this work, however, the performance of a firstorder filter was found to be satisfactory.

\section{APPENDIX C: SNR ESTIMATION}

SNR estimation is a nonconsensual topic, and several different definitions and approaches are commonly used. Our method for estimating SNR with recursive channel identification parallels that of Flynn et al. ${ }^{17}$ for block-based estimation. Specifically, we concentrate on an approach that tries to include reverberation in the total noise present during packet reception, as it cannot be coherently combined at the receiver to improve symbol estimates when using identification-based algorithms (equalization and TRM with probe estimation).

Let the samples of the $m$ th estimated pulse shape $h_{m}(t)$ in Eq. (1), oversampled by a factor of $L$, be collected in vector $\mathbf{h}_{m}$. As described in Sec. IV B, the $L$ polyphase components of $h_{m}$ can be separately identified from a common training sequence and the polyphase components of the received signal $y_{m}$ using a parallel bank of RLS filters, whose residual MSE is theoretically identical and will be denoted by $\sigma_{m \text { id. }}^{2}$. The variance $\sigma_{m \text { id }}^{2}$ includes not only power from physical disturbances, $\sigma_{m d}^{2}$ (ambient noise and reverbera- tion), but also excess MSE, $\zeta_{m}$, due to misadjustment between actual channel coefficients and those estimated by the adaptive identification algorithm

$$
\sigma_{m \text { id }}^{2}=\sigma_{m d}^{2}+\zeta_{m}, \quad \sigma_{m d}^{2} \triangleq \sigma_{m \mathrm{amb}}^{2}+\sigma_{m \text { rev }}^{2} .
$$

Similarly, the expected value of the coefficient vector norm $\left\|\mathbf{h}_{m}\right\|^{2}$ (useful signal energy) exceeds the true norm by the trace of its covariance matrix. Approximate closed-form expressions are available for the variance of these estimation errors in several adaptive algorithms. ${ }^{30}$ For RLS operating with forgetting factor $\lambda$ and $N$ coefficients that provide enough degrees of freedom to model the underlying system, the output MSE is ${ }^{30}$

$$
\sigma_{m d}^{2}+\zeta_{m}=\frac{2 \sigma_{m d}^{2}+\frac{\gamma_{m}^{2}}{1-\lambda}}{2-(1-\lambda) N},
$$

where the constant $\gamma_{m}$ is related to the degree of nonstationarity of the system. One possible approach for estimating $\sigma_{m d}^{2}$ is to empirically evaluate the residual MSE $\sigma_{m \text { id }}^{2}$ for a range of values of $\lambda$ and fit that curve to the right-hand side of Eq. (C2) to obtain the unknown $\sigma_{m d}^{2}$ (and $\gamma_{m}^{2}$ as a byproduct). Note that the theoretical derivation of Eq. (C2) assumes $\lambda \approx 1$ and $(1-\lambda) N \ll 1$, neither of which are very accurate for the values of $\lambda$ and $N$ where the lowest empirical $\sigma_{m d}^{2}$ is obtained in MREA'04 data.

The average SINR is defined as ${ }^{17}$

$$
\operatorname{SINR}=\frac{1}{M} \sum_{m=1}^{M} \frac{\left\|\mathbf{h}_{m}\right\|^{2}}{\sigma_{m}^{2}{ }_{d} L} .
$$

Technically, $\left\|\mathbf{h}_{m}\right\|^{2}$ in Eq. (C3) should be fine-tuned by subtracting the excess norm due to RLS adaptation. This was deemed unnecessary given the coarseness of approximations in the estimation of $\sigma_{m d}^{2}$. In summary, the following steps were carried out to estimate SINR for each packet.

(1) Estimate the channel response in each array sensor as described in Sec. IV B for a range of RLS forgetting factors $\lambda_{\min } \leqslant \lambda_{i} \leqslant \lambda_{\max }, i=1, \ldots, F$.

(2) Compute the steady-state MSE $\sigma_{m}^{2}$ id at the filter output for each $\lambda_{i}$, averaging across polyphase components. Collect these in an $F \times 1$ vector $\mathbf{b}_{m}$.

(3) Build an $F \times 2$ matrix $\mathbf{A}$ whose $i$ th row $\mathbf{a}_{i}$ equals

$$
\mathbf{a}_{i}=\frac{1}{2-\left(1-\lambda_{i}\right) N}\left[\begin{array}{ll}
2 & \frac{1}{1-\lambda_{i}}
\end{array}\right],
$$

where $N$ is the order of the identification filter per polyphase component.

(4) Solve $\mathbf{A} \mathbf{x}_{m}=\mathbf{b}_{m}$ in the least-squares sense to get $\mathbf{x}_{m}$ $=\left[\begin{array}{ll}\sigma_{m d}^{2} \gamma_{m}^{2}\end{array}\right]^{T}$.

(5) Compute the SINR according to Eq. (C3), where $\mathbf{h}_{m}$ is the $L$-oversampled identified channel response for $\lambda_{i}$ where the smallest identification residual $\sigma_{m}^{2}$ id was obtained.

Finally, note that throughout this work TRM performance is 
expressed in terms of output MSE. Most authors who prefer to use a $\mathrm{SNR}_{\text {out }}$ metric ${ }^{6,11,17}$ essentially adopt a simple sign change in decibel scale, $\mathrm{SNR}_{\mathrm{out}} \approx \mathrm{MSE}^{-1}$.

${ }^{1}$ D. R. Jackson and D. R. Dowling, "Phase conjugation in underwater acoustics," J. Acoust. Soc. Am. 89, 171-181 (1991).

${ }^{2}$ W. A. Kuperman, W. S. Hodgkiss, H. C. Song, T. Akal, C. Ferla, and D. R. Jackson, "Phase conjugation in the ocean: Experimental demonstration of an acoustic time-reversal mirror," J. Acoust. Soc. Am. 103, 25-40 (1998). ${ }^{3}$ D. R. Dowling, "Acoustic pulse compression using passive phaseconjugate processing," J. Acoust. Soc. Am. 95, 1450-1458 (1994).

${ }^{4}$ M. Stojanovic, J. A. Catipovic, and J. G. Proakis, "Reduced-complexity spatial and temporal processing of underwater acoustic communication signals," J. Acoust. Soc. Am. 98, 961-972 (1995).

${ }^{5}$ M. Fink, "Time-reversed acoustics," Sci. Am. 281(5), 91-97 (1999).

${ }^{6} \mathrm{M}$. Stojanovic, "Retrofocusing techniques for high rate acoustic communications," J. Acoust. Soc. Am. 117, 1173-1185 (2005).

${ }^{7}$ G. F. Edelmann, T. Akal, W. S. Hodgkiss, S. Kim, W. A. Kuperman, and H. C. Song, "An initial demonstration of underwater acoustic communication using time reversal," IEEE J. Ocean. Eng. 27, 602-609 (2002).

${ }^{8}$ G. F. Edelmann, H. C. Song, S. Kim, W. S. Hodgkiss, W. A. Kuperman, and T. Akal, "Underwater acoustic communications using time reversal," IEEE J. Ocean. Eng. 30, 852-864 (2005).

${ }^{9}$ D. Rouseff, D. R. Jackson, W. L. J. Fox, C. D. Jones, J. A. Ritcey, and D. R. Dowling, "Underwater acoustic communication by passive-phase conjugation: Theory and experimental results," IEEE J. Ocean. Eng. 26, 821831 (2001).

${ }^{10}$ A. Silva, S. Jesus, J. Gomes, and V. Barroso, "Underwater acoustic communication using a "virtual' electronic time-reversal mirror approach," Proceedings of the V European Conference on Underwater Acoustics (ECUA'00), edited by P. Chevret and M. E. Zakharia, Lyon, France, 2000. ${ }^{11}$ H. C. Song, W. S. Hodgkiss, W. A. Kuperman, W. J. Higley, K. Raghukumar, T. Akal, and M. Stevenson, "Spatial diversity in passive time reversal communications," J. Acoust. Soc. Am. 120, 2067-2076 (2006).

${ }^{12}$ H. C. Song, W. S. Hodgkiss, W. A. Kuperman, M. Stevenson, and T. Akal, "Improvement of time-reversal communications using adaptive channel equalizers," IEEE J. Ocean. Eng. 31, 487-496 (2006).

${ }^{13} \mathrm{D}$. Rouseff, "Intersymbol interference in underwater acoustic communications using time-reversal signal processing," J. Acoust. Soc. Am. 117, 780-788 (2005).

${ }^{14}$ P. Hursky, M. B. Porter, M. Siderius, and V. K. McDonald, "Point-to-point underwater acoustic communications using spread-spectrum passive phase conjugation," J. Acoust. Soc. Am. 120, 247-257 (2006).

${ }^{15}$ H. C. Song, P. Roux, W. S. Hodgkiss, W. A. Kuperman, T. Akal, and M. Stevenson, "Multiple-input-multiple-output coherent time reversal communications in a shallow-water acoustic channel," IEEE J. Ocean. Eng. 31, 170-178 (2006).

${ }^{16} \mathrm{~J}$. Gomes and V. Barroso, "Asymmetric underwater acoustic communication using a time-reversal mirror," Proceedings of MTS/IEEE OCEANS'00, Providence, RI, 2000, Vol. 3, 1847-1851.
${ }^{17}$ J. A. Flynn, J. A. Ritcey, D. Rouseff, and W. L. J. Fox, "Multichannel equalization by decision-directed passive phase conjugation: Experimental results," IEEE J. Ocean. Eng. 29, 824-836 (2004).

${ }^{18}$ J. G. Proakis, Digital Communications, 4th ed. (McGraw-Hill, New York, 2000).

${ }^{19}$ T. C. Yang, "Temporal resolution of time-reversal and passive phaseconjugation processing,” IEEE J. Ocean. Eng. 28, 229-245 (2003).

${ }^{20}$ L. J. Ziomek, Fundamentals of Acoustic Field Theory and Space-Time Signal Processing (CRC, Boca Raton, FL, 1995).

${ }^{21}$ H. C. Song, W. A. Kuperman, and W. S. Hodgkiss, "A time-reversal mirror with variable range focusing," J. Acoust. Soc. Am. 103, 3234-3240 (1998).

${ }^{22}$ S. Kim, W. A. Kuperman, W. S. Hodgkiss, H. C. Song, G. F. Edelmann, and T. Akal, "Robust time reversal focusing in the ocean," J. Acoust. Soc. Am. 114, 145-157 (2003).

${ }^{23} \mathrm{H}$. Cox, "Navy applications of high frequency acoustics," Proceedings of the High-Frequency Ocean Acoustics Conference (HFOAC'04), La Jolla, CA, 2004, Vol. 728, 449-455.

${ }^{24} \mathrm{P}$. Balaban and J. Salz, "Optimum diversity combining and equalization in digital data transmission with applications to cellular mobile radio-Part I: Theoretical considerations," IEEE Trans. Commun. 40, 885-894 (1992).

${ }^{25}$ M. Stojanovic, J. A. Catipovic, and J. G. Proakis, "Adaptive multichannel combining and equalization for underwater acoustic communications," J. Acoust. Soc. Am. 94, 1621-1631 (1993).

${ }^{26}$ A. Parvulescu, "Matched-signal ("MEss") processing by the ocean," J. Acoust. Soc. Am. 98, 943-960 (1995).

${ }^{27}$ M. B. Porter and H. P. Bucker, "Gaussian beam tracing for computing ocean acoustic fields," J. Acoust. Soc. Am. 82, 1349-1359 (1987).

${ }^{28} \mathrm{O}$. Shalvi and E. Weinstein, "New criteria for blind deconvolution of nonminimum phase systems (channels)," IEEE Trans. Inf. Theory 36, 312321 (1990).

${ }^{29}$ J. Gomes, A. Silva, and S. Jesus, "Joint passive time reversal and multichannel equalization for underwater communications," Proceedings of MTS/IEEE OCEANS'06, Boston, MA, 2006, 1161-1166.

${ }^{30}$ A. H. Sayed, Fundamentals of Adaptive Filtering (Wiley-IEEE, New York, 2003).

${ }^{31}$ S. Jesus, C. Soares, P. Felisberto, A. Silva, L. Farinha, and C. Martins, "Acoustic maritime rapid environmental assessment during the MREA'04 sea trial," Technical Report No. 02/05, Centro de Investigacao Tecnologica do Algarve, Universidade do Algarve, 2005, URL: ftp://ftp.ualg.pt/users/ sjesus/pubs/B21.pdf, accessed on 6/28/2008

${ }^{32} \mathrm{~S}$. Ariyavisitakul and L. J. Greenstein, "Reduced-complexity equalization techniques for broadband wireless channels," IEEE J. Sel. Areas Commun. 15, 5-15 (1997).

${ }^{33} \mathrm{~J}$. C. Preisig, "Performance analysis of adaptive equalization for coherent acoustic communications in the time-varying ocean environment," J. Acoust. Soc. Am. 118, 263-278 (2002).

${ }^{34}$ B. S. Sharif, J. Neasham, O. R. Hinton, and A. E. Adams, "A computationally efficient doppler compensation system for underwater acoustic communications," IEEE J. Ocean. Eng. 25, 52-61 (2000). 